\title{
Review
}

\section{A Critical Review of the Pharmacokinetics and Pharmacodynamics of Opioid Medications Used in Avian Patients}

\author{
Evelyn Aliansyah ${ }^{1}$, Hui Ting Chng ${ }^{1, *(\mathbb{D})}$ and Shangzhe Xie ${ }^{2, *(\mathbb{D}}$ \\ 1 Department of Pharmacy, National University of Singapore, 18 Science Drive 4 Blk S4A Level 3, \\ Singapore 117559, Singapore; evelynaliansyah@u.nus.edu \\ 2 Mandai Wildlife Group, Conservation, Research and Veterinary Department, 80 Mandai Lake Road, \\ Singapore 729826, Singapore \\ * Correspondence: phacht@nus.edu.sg (H.T.C.); shangzhe.xie@wrs.com.sg (S.X.)
}

check for updates

Citation: Aliansyah, E.; Chng, H.T.; Xie, S. A Critical Review of the Pharmacokinetics and

Pharmacodynamics of Opioid

Medications Used in Avian Patients. Birds 2022, 3, 1-28. https:/ /doi.org /10.3390/birds3010001

Academic Editor: Jukka Jokimäki

Received: 22 June 2021

Accepted: 20 December 2021

Published: 23 December 2021

Publisher's Note: MDPI stays neutral with regard to jurisdictional claims in published maps and institutional affiliations.

Copyright: (C) 2021 by the authors. Licensee MDPI, Basel, Switzerland. This article is an open access article distributed under the terms and conditions of the Creative Commons Attribution (CC BY) license (https:// creativecommons.org/licenses/by/ $4.0 /)$.
Simple Summary: In administering opioid medications for a particular species of bird, doses are usually extrapolated from other species of birds. This is unideal as differences in the physiology of birds, even among closely related species, may affect drug action and disposition, and hence the efficacy and safety of drugs used. We collated opioid dosing regimens used in birds from the literature and evaluated their efficacy, safety and pharmacokinetic data. Tramadol, hydromorphone, buprenorphine, butorphanol and fentanyl were found to be generally safe. However, there was a lack of analgesic effects for hydromorphone and buprenorphine in Cockatiels (Nymphicus hollandicus). American Kestrels (Falco sparverius) appeared to exhibit sex-dependent responses to opioids. Differences in the bioavailability of oral tramadol was seen between Hispaniolan Parrot (Amazona ventralis) and Bald Eagle (Haliaeetus leucocephalus).

\begin{abstract}
Opioid drugs are used to manage moderate to severe pain in mammals and avian species. In dosing opioids for a particular species, it is optimal to use dosing regimens based on pharmacokinetics or pharmacodynamics studies conducted in the same species as variability in the physiology among different species may result in differences in drug pharmacokinetics and pharmacodynamics. Unfortunately, dosing regimens are typically extrapolated from closely related avian species or even mammals, which is unideal. Therefore, this critical review aims to collate and evaluate the dosing regimens of selected opioids: tramadol, hydromorphone, buprenorphine, butorphanol, and fentanyl, in avian species and its related safety, efficacy and pharmacokinetic data. Our review found specific dosing regimens not described in the Exotic Animal Formulary for tramadol used in Indian Peafowl (Pavo cristatus), Muscovy Duck (Cairina moschata) and Hispaniolan Parrot (Amazona ventralis); hydromorphone used in Orange-winged Parrot (Amazona amazonica); buprenorphine used in Cockatiel (Nymphicus hollandicus), American Kestrel (Falco sparverius) and Grey Parrot (Psittacus erithacus); and butorphanol used in Hispaniolan Parrot (Amazona ventralis), Broiler Chicken and Indian Peafowl (Pavo cristatus). Cockatiel appeared to not experience analgesic effects for hydromorphone and buprenorphine, and American Kestrel exhibited sex-dependent responses to opioids. The selected opioids were observed to be generally safe, with adverse effects being dose-dependent.
\end{abstract}

Keywords: opioids; tramadol; hydromorphone; buprenorphine; butorphanol; fentanyl; birds; avian; pharmacokinetics; dosing

\section{Introduction}

There are 10,806 extant species of birds in the world, which are classified to 40 orders, 252 families and 2353 genera [1]. 20.6 million birds were owned as companion in the United States households and 6\% of pet owners in Singapore owned pet birds in 2017 and 2018, respectively [2,3], making birds important for their sentimental value to humans. Birds are also important as they are featured in zoos and parks, as well as for their value in nature conservation. 
In efforts to conserve bird species or keeping them as pets, birds may experience potentially painful injuries, diseases or surgical procedures. Alleviating pain in birds may not be as straightforward as doing so in humans as they are unable to express pain effectively through facial or verbal expressions. There could also be situations where birds potentially experience pain, but do not show any behavioral or facial changes. Even when behavioral changes in birds potentially experiencing pain are present, they could still be difficult to assess as this requires the observer to be able to recognize the full range of normal and abnormal behaviors of each species and individual to assess pain. There are also other factors to be considered when assessing pain in birds, including age, species, gender, environment, strain, and breed [4,5]. Therefore, if any of the points above hold true, veterinarians must assume that the animals are in pain and development of an analgesic plan is deemed necessary if:

(A) The lesion or procedure would be painful to other species;

(B) The lesion or procedure is damaging to tissues in other species;

(C) The patient shows any abnormal behaviour [4].

Providing appropriate analgesia is an important aspect of veterinary care in all species, including avian species, that are going through potentially painful experiences or procedures. Other than the moral obligation to provide analgesic plans in managing potentially painful injuries in birds, there are also other reasons to manage pain in birds. Injury may cause an animal to be immobilized, resulting in muscle weakness and wasting [6]. Arthritisinduced Muscovy Duck (Cairina moschata) in the control arm had a greater difference in maximum force on left and right legs as compared to those treated with pain-relieving drugs such as meloxicam and tramadol, indicating lameness on the arthritis-induced leg [7]. This indicates that relieving pain is important in maintaining mobility of birds. Furthermore, poorly treated pain can result in self-mutilation or lead to chronic pain syndromes. Birds may resort to damaging behaviors such as feather picking [8]. This may result in reduced aesthetic in birds, reduced ability to keep themselves warm and dry, as well as an increased exposure to skin infections or other complications [8,9].

Opioid drugs, in veterinary medicine, are used for moderate to severe pain such as in fractures or surgery [4]. Generally, the Exotic Animal Formulary (EAF) is used as a reference for dosing regimen of specific opioid drugs [10]. However, there is a lack of dosing regimens of opioids in selected species of birds. Thus, when data is unavailable for selected species of birds, doses are often extrapolated from closely-related bird species or other animal species [11-14]. However, interspecies variability in terms of the anatomy and physiology is present even between closely-related bird species. This may translate to differences in drug pharmacokinetics (PK) and/or pharmacodynamics (PD), resulting in differences in the safety and efficacy, when the same drug dosing regimen is administered across different species [11]. For example, in studies evaluating the analgesic effects of butorphanol using the isoflurane-sparing technique, $1 \mathrm{mg} / \mathrm{kg}$ of butorphanol was found to be analgesic in Cockatoos (Cacatua galerita, Cacatua sulphurea cintrinocristata, Cacatua sulphurea sulphurea), but not Hispaniolan Parrot (Amazona ventralis) [5] These results highlight variations in drug PK and PD between species, even among the closely-related psittacine species. Therefore, intraspecies scaling of dosing regimen in a particular species of birds is unideal [12-14].

In mammals, analgesia is achieved when opioids bind to either $\mu-, \mathrm{K}_{-}$, or $\delta$-opioid receptors in the central nervous system (CNS), either spinally or supraspinally [4]. The opioids are categorized as agonists, partial agonists, mixed agonist/antagonists or antagonists. This categorization depends on each opioid's ability to induce analgesic response when bound to a specific receptor. The agonists have a linear dose-response curve, whereby dose can be increased to achieve the desired effect. On the other hand, mixed agonist/antagonist opioids may have agonist property to one receptor but act as antagonist at another type of receptor. Agonist/antagonist opioids may reach a plateau in terms of its analgesic effect, where increasing the dose does not provide additional analgesia [15].

Besides analgesia, opioids are often used in mammals during anesthesia to provide the anesthesia-sparing effect, where use of opioids may reduce the concentration of volatile 
anesthetics required [6]. In birds, opioids can also be used to provide the anesthesia-sparing effect [16-18].

Opioid analgesics commonly used in birds are those that act on $\mu$ - or k-opioid receptors. This critical review will discuss these opioids, particularly on tramadol, hydromorphone, buprenorphine, butorphanol and fentanyl. Table 1 summarizes the interactions of selected opioids with $\mu$ - or k-opioid receptors.

Table 1. The interactions of selected opioids with $\mu$ - or k-opioid receptors and their classification.

\begin{tabular}{ccc}
\hline Opioid & $\begin{array}{c}\text { Interaction with } \\
\mu \text {-Opioid Receptor }\end{array}$ & $\begin{array}{c}\text { Interaction with } \\
\text { K-Opioid Receptors }\end{array}$ \\
\hline Tramadol [19] & Weak agonist & - \\
\hline Hydromorphone [20] & Agonist & - \\
\hline Buprenorphine [21] & Partial agonist-antagonist & - \\
\hline Butorphanol [22] & Mixed agonist/antagonist & Partial agonist \\
\hline Fentanyl [23] & Agonist & - \\
\hline
\end{tabular}

Therefore, this critical review aims to collate evidence of the dosing regimens of opioids used in different avian species, evaluate their safety and efficacy data, and identify any trends in the PK of the opioids in the different avian species.

\section{Methods}

Articles were searched on PubMed (https://pubmed.ncbi.nlm.nih.gov/) accessed on 30 January 2021 using keywords related to the concept of birds, opioids, and pharmacokinetic (PK) and pharmacodynamic (PD). The full list of keywords can be found in Table A1. Efficacy and adverse effects were not searched for, but were recorded if data was found in the included papers. For a paper to be included, it had to provide either PK or PD information on the opioid used in particular species of birds. PD information is related to how the bird reacts to antinociceptive stimulus such as thermal or electrical. Data extracted included: name of species, weight and age of species, sample size, dosing regimen, half-life, $C_{\max }$, bioavailability, duration mean plasma concentration exceeding target plasma concentration considered analgesic in other animals/human, adverse effects and dosing recommendation.

\section{Overview of Dosing Regimens of Opioids Used in Avian Species}

This section provides information on dosing regimens of selected opioids studied in various species of birds. It also identifies dosing regimens not found in the EAF 5th edition. Dosing regimens recommended by EAF are generally dosing regimens that have been studied or are subsets of what was studied. However, there were a few exceptions whereby the dosing regimens recommended by EAF were higher or lower than the dosage studied, which will be highlighted in Table 2. Specific dosing regimens not described in EAF was found for tramadol used in Indian Peafowl (Pavo cristatus), Muscovy Duck (Cairina moschata) and Hispaniolan Parrot (Amazona ventralis); hydromorphone used in Orange-winged Parrot (Amazona amazonica); buprenorphine used in Cockatiel (Nymphicus hollandicus), American Kestrel (Falco sparverius) and Grey parrot (Psittacus Erithacus); and butorphanol used in Hispaniolan Parrot (Amazona ventralis), Broiler Chicken (Gallus gallus domesticus) and Indian Peafowl (Pavo cristatus). The details of the dosing regimens are shown in Table 2. 
Table 2. Dosing regimens of selected opioids studied in various species of birds.

\begin{tabular}{|c|c|c|c|c|c|c|c|c|}
\hline Drug & $\begin{array}{c}\text { Species } \\
\text { (English Name) }\end{array}$ & $\begin{array}{c}\text { Species } \\
\text { (Scientific Name) }\end{array}$ & $\begin{array}{c}\text { Route of } \\
\text { Administration }\end{array}$ & $\underset{\text { (Years) }}{\text { Age }}$ & $\begin{array}{l}\text { Duration of } \\
\text { Treatment }\end{array}$ & $\begin{array}{l}\text { Dosing } \\
\text { Regimen } \\
\text { Studied }\end{array}$ & $\begin{array}{c}\text { Dosing Regimen } \\
\text { Found in Exotic } \\
\text { Animal } \\
\text { Formulary 5th } \\
\text { Edition (Yes/No) }\end{array}$ & Reference \\
\hline \multirow{9}{*}{ Tramadol } & \multirow[t]{2}{*}{ Bald Eagle } & \multirow{2}{*}{$\begin{array}{c}\text { Haliaeetus } \\
\text { leucocephalus }\end{array}$} & Oral & - & Single dose & $11 \mathrm{mg} / \mathrm{kg}^{1}$ & \multirow{2}{*}{ Yes } & \multirow{2}{*}[24,25]{} \\
\hline & & & Intravenous & - & Single dose & $4 \mathrm{mg} / \mathrm{kg}^{2}$ & & \\
\hline & American Kestrel & Falco sparverius & Oral & 3 & Single dose & $\begin{array}{c}5 \mathrm{mg} / \mathrm{kg} \\
15 \mathrm{mg} / \mathrm{kg} \\
30 \mathrm{mg} / \mathrm{kg}\end{array}$ & Yes & [26] \\
\hline & Red-tailed Hawk & Buteo jamaicensis & Oral & - & Single dose & $11 \mathrm{mg} / \mathrm{kg}^{3}$ & Yes $^{4}$ & {$[25,27]$} \\
\hline & Jackass Penguin & $\begin{array}{l}\text { Spheniscus } \\
\text { demersus }\end{array}$ & Oral & $1.5-20$ & Single dose & $10 \mathrm{mg} / \mathrm{kg}$ & Yes & [28] \\
\hline & \multirow{2}{*}{ Hispaniolan Parrot } & \multirow{2}{*}{ Amazona ventralis } & Oral & - & $\begin{array}{l}\text { Repeated dose } \\
\text { (BD) for } 5 \text { days }\end{array}$ & $30 \mathrm{mg} / \mathrm{kg}$ & Yes & \multirow{2}{*}{ [29-32] } \\
\hline & & & Intravenous & - & Single dose & $5 \mathrm{mg} / \mathrm{kg}$ & No & \\
\hline & Indian Peafowl & Pavo cristatus & Oral & - & Single dose & $7.5 \mathrm{mg} / \mathrm{kg}$ & No & [33] \\
\hline & Muscovy Duck & Cairina moschata & Oral & - & Single dose & $30 \mathrm{mg} / \mathrm{kg}$ & No & {$[7,34]$} \\
\hline \multirow{4}{*}{ Hydromorphone } & American Kestrel & Falco sparverius & Intramuscular & 2 & Single dose & $\begin{array}{l}0.1 \mathrm{mg} / \mathrm{kg} \\
0.3 \mathrm{mg} / \mathrm{kg} \\
0.6 \mathrm{mg} / \mathrm{kg}\end{array}$ & Yes & {$[35,36]$} \\
\hline & Cockatiel & $\begin{array}{l}\text { Nymphicus } \\
\text { hollandicus }\end{array}$ & Intramuscular & $2-6$ & Single dose & $\begin{array}{l}0.1 \mathrm{mg} / \mathrm{kg} \\
0.3 \mathrm{mg} / \mathrm{kg} \\
0.6 \mathrm{mg} / \mathrm{kg}\end{array}$ & Yes & [37] \\
\hline & \multirow[t]{2}{*}{ Orange-winged Parrot } & \multirow[t]{2}{*}{$\begin{array}{l}\text { Amazona } \\
\text { amazonica }\end{array}$} & Intramuscular & $\begin{array}{c}4-17 \\
\text { and } \\
28-45\end{array}$ & Single dose & $\begin{array}{l}0.1 \mathrm{mg} / \mathrm{kg} \\
1 \mathrm{mg} / \mathrm{kg} \\
2 \mathrm{mg} / \mathrm{kg}\end{array}$ & $\mathrm{No}^{5}$ & \multirow[t]{2}{*}[38,39]{} \\
\hline & & & Intravenous & $28-45$ & Single dose & $1 \mathrm{mg} / \mathrm{kg}$ & No & \\
\hline \multirow{10}{*}{ Buprenorphine } & \multirow{2}{*}{ Grey Parrot } & \multirow{2}{*}{ Psittacus erithacus } & Intramuscular & - & Single dose & $0.1 \mathrm{mg} / \mathrm{kg}$ & Yes $^{6}$ & \multirow{2}{*}[40,41]{} \\
\hline & & & Intravenous & - & Single dose & $0.1 \mathrm{mg} / \mathrm{kg}$ & No & \\
\hline & \multirow{4}{*}{ American Kestrel } & \multirow{4}{*}{ Falco sparverius } & Intramuscular & 3 & Single dose & $\begin{array}{l}0.1 \mathrm{mg} / \mathrm{kg} \\
0.3 \mathrm{mg} / \mathrm{kg} \\
0.6 \mathrm{mg} / \mathrm{kg}\end{array}$ & Yes & \multirow[t]{2}{*}[42,43]{} \\
\hline & & & Intravenous & 3 & Single dose & $0.6 \mathrm{mg} / \mathrm{kg}$ & No & \\
\hline & & & $\begin{array}{l}\text { Subcutenous } \\
\text { (sustained-release) }\end{array}$ & $3-5$ & Single dose & $1.8 \mathrm{mg} / \mathrm{kg}$ & Yes & \multirow{2}{*}[44,45]{} \\
\hline & & & $\begin{array}{l}\text { Intramuscular } \\
\text { (sustained-release) }\end{array}$ & $3-5$ & Single dose & $1.8 \mathrm{mg} / \mathrm{kg}$ & Yes & \\
\hline & \multirow[b]{2}{*}{ Red-tailed Hawk } & \multirow[b]{2}{*}{ Buteo jamaicensis } & Intramuscular & - & Twice daily & $0.25 \mathrm{mg} / \mathrm{kg}$ & Yes & [46] \\
\hline & & & $\begin{array}{l}\text { Subcutaneous } \\
\text { (concentrated) }\end{array}$ & - & Single dose & $\begin{array}{l}0.3 \mathrm{mg} / \mathrm{kg} \\
1.8 \mathrm{mg} / \mathrm{kg}\end{array}$ & Yes & [47] \\
\hline & Rock Dove & Columba livia & Intramuscular & - & - & $\begin{array}{l}\text { Unable to find } \\
\text { evidence }\end{array}$ & Yes & [10] \\
\hline & Cockatiel & $\begin{array}{l}\text { Nymphicus } \\
\text { hollandicus }\end{array}$ & Intramuscular & $2-6$ & Single dose & $\begin{array}{l}0.6 \mathrm{mg} / \mathrm{kg} \\
1.2 \mathrm{mg} / \mathrm{kg} \\
1.8 \mathrm{mg} / \mathrm{kg} \\
\end{array}$ & $\mathrm{No}^{7}$ & [48] \\
\hline \multirow{11}{*}{ Butorphanol } & \multirow{5}{*}{ Hispaniolan Parrot } & \multirow{5}{*}{ Amazona ventralis } & Oral & 8 & Single dose & $5 \mathrm{mg} / \mathrm{kg}$ & No & \multirow{2}{*}{ [49] } \\
\hline & & & Intravenous & 8 & Single dose & $5 \mathrm{mg} / \mathrm{kg}$ & Yes & \\
\hline & & & Intramuscular & 8 & Single dose & $\begin{array}{c}2 \mathrm{mg} / \mathrm{kg}^{8} \\
5 \mathrm{mg} / \mathrm{kg}\end{array}$ & Yes & {$[49,50]$} \\
\hline & & & $\begin{array}{l}\text { Subcutaneous } \\
\text { (long-acting) }\end{array}$ & - & Single dose & $12 \mathrm{mg} / \mathrm{kg}$ & No & [51] \\
\hline & & & $\begin{array}{l}\text { Subcutaneous } \\
\text { (liposome- } \\
\text { encapsulated) }\end{array}$ & $11-27$ & Single dose & $\begin{array}{l}10 \mathrm{mg} / \mathrm{kg} \\
15 \mathrm{mg} / \mathrm{kg}\end{array}$ & No & {$[50,52]$} \\
\hline & $\begin{array}{l}\text { Grey Parrot and } \\
\text { Timneh Parrot }\end{array}$ & $\begin{array}{l}\text { Psittacus erithacus } \\
\text { and Psittacus } \\
\text { timneh }\end{array}$ & Intramuscular & - & Single dose & $1 \mathrm{mg} / \mathrm{kg}$ & Yes & [41] \\
\hline & \multirow{2}{*}{ Red-tailed Hawk } & & Intravenous & - & Single dose & $0.5 \mathrm{mg} / \mathrm{kg}$ & Yes & {$[50]$} \\
\hline & & Buteo jamaicensis & Intramuscular & - & Single dose & $0.5 \mathrm{mg} / \mathrm{kg}$ & Yes & [53] \\
\hline & & 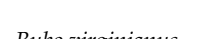 & Intravenous & - & Single dose & $0.5 \mathrm{mg} / \mathrm{kg}$ & Yes & {$[52]$} \\
\hline & Great Horned Uw1 & Биюo oirgintanus & Intramuscular & - & Single dose & $0.5 \mathrm{mg} / \mathrm{kg}$ & Yes & [30] \\
\hline & American Kestrel & Falco sparverius & Intramuscular & $2-3$ & Single dose & $\begin{array}{l}1 \mathrm{mg} / \mathrm{kg} \\
3 \mathrm{mg} / \mathrm{kg} \\
6 \mathrm{mg} / \mathrm{kg}\end{array}$ & Yes & [54] \\
\hline
\end{tabular}


Table 2. Cont.

\begin{tabular}{|c|c|c|c|c|c|c|c|c|}
\hline Drug & $\begin{array}{c}\text { Species } \\
\text { (English Name) }\end{array}$ & $\begin{array}{c}\text { Species } \\
\text { (Scientific Name) }\end{array}$ & $\begin{array}{c}\text { Route of } \\
\text { Administration }\end{array}$ & $\underset{\text { (Years) }}{\text { Age }}$ & $\begin{array}{l}\text { Duration of } \\
\text { Treatment }\end{array}$ & $\begin{array}{l}\text { Dosing } \\
\text { Regimen } \\
\text { Studied }\end{array}$ & $\begin{array}{c}\text { Dosing Regimen } \\
\text { Found in Exotic } \\
\text { Animal } \\
\text { Formulary 5th } \\
\text { Edition (Yes/No) }\end{array}$ & Reference \\
\hline & $\begin{array}{l}\text { Sulphur-crested } \\
\text { Cockatoo and } \\
\text { Yellow-crested } \\
\text { Cockatoo }\end{array}$ & $\begin{array}{l}\text { Cacatua galerita, } \\
\text { Cacatua sulphurea } \\
\text { citrinocristata and } \\
\text { Cacatua sulphurea } \\
\text { sulphurea }\end{array}$ & Intramuscular & - & Single dose & $1 \mathrm{mg} / \mathrm{kg}$ & Yes & [16] \\
\hline & Psittacine & - & $\begin{array}{l}\text { Constant-Rate } \\
\text { Infusion }\end{array}$ & - & - & $\begin{array}{l}\text { Unable to } \\
\text { find evidence }\end{array}$ & Yes $^{9}$ & [10] \\
\hline & Ratite & - & Intravenous & - & - & $\begin{array}{c}\text { Unable to } \\
\text { find evidence }\end{array}$ & Yes ${ }^{10}$ & {$[10]$} \\
\hline & Broiler Chicken & - & Intravenous & - & Single dose & $2 \mathrm{mg} / \mathrm{kg}$ & No & [55] \\
\hline & Indian Peafowl & Pavo cristatus & Osmotic pump & - & 7 continuous days & $\begin{array}{c}120 \mu \mathrm{g} / \mathrm{kg} / \mathrm{hr} \\
\text { with rate } 48.1 \\
\mathrm{mg} / \mathrm{mL}^{11}\end{array}$ & No & {$[56]$} \\
\hline \multirow{6}{*}{ Fentanyl } & \multirow{2}{*}{$\begin{array}{l}\text { African and New } \\
\text { World Parrots }\end{array}$} & \multirow{2}{*}{ Cacatua alba } & Intramuscular & - & Single dose & $\begin{array}{l}0.01 \mathrm{mg} / \mathrm{kg} \\
0.02 \mathrm{mg} / \mathrm{kg}\end{array}$ & Yes & \multirow[t]{2}{*}[57]{} \\
\hline & & & Subcutaneous & - & Single dose & $0.2 \mathrm{mg} / \mathrm{kg}$ & Yes $^{12}$ & \\
\hline & Red-tailed Hawk & Buteo jamaicensis & $\begin{array}{l}\text { Constant-Rate } \\
\text { Infusion }\end{array}$ & - & - & $\begin{array}{l}\text { Unable to find } \\
\text { evidence }^{13}\end{array}$ & Yes & {$[10]$} \\
\hline & Hispaniolan Parrot & Amazona ventralis & $\begin{array}{l}\text { Constant-Rate } \\
\text { Infusion }\end{array}$ & - & - & $\begin{array}{l}\text { Unable to find } \\
\text { evidence }{ }^{14}\end{array}$ & Yes & {$[10]$} \\
\hline & Helmeted Guineafowl & Numida meleagris & Transdermal & - & Single dose & $5 \mathrm{mg} / \mathrm{kg}$ & No & [58] \\
\hline & $\begin{array}{c}\text { Chickens (Brahma, } \\
\text { Delaware, Redstar and } \\
\text { Wyandotte) }\end{array}$ & - & Transdermal & 3 & Single dose & $\begin{array}{c}25 \mu \mathrm{g} / \mathrm{h} \text { for } 72 \\
\mathrm{~h}\end{array}$ & No & [59] \\
\hline
\end{tabular}

${ }^{1}$ Dosing regimen recommended by both author and Exotic Animal Formulary 5 th edition is $5 \mathrm{mg} / \mathrm{kg} \mathrm{q} 12 \mathrm{~h}$ Recommendation is based on simulation in the stud. ${ }^{2}$ Dosing regimen recommended in Exotic Animal Formulary 5th edition is $5 \mathrm{mg} / \mathrm{kg}$, which is higher than dosage studied. ${ }^{3}$ Dosing regimen recommended by author is $15 \mathrm{mg} / \mathrm{kg} \mathrm{q} 12 \mathrm{~h}$. Recommendation is based on simulation. ${ }^{4}$ Dosing regimen recommended in Exotic Animal Formulary 5th edition is $8-11 \mathrm{mg} / \mathrm{kg} .{ }^{5}$ Exotic Animal Formulary 5th edition was available online in November 2017 but Pharmacokinetics and Pharmacodynamics papers were published in $2020 .{ }^{6}$ Dosing regimen in Exotic Animal Formulary 5 th edition also includes $0.25 \mathrm{mg} / \mathrm{kg}$, which is not studied. ${ }^{7}$ Paper was published in December 2018 while Exotic Animal Formulary 5th edition was available online in November $2017 .{ }^{8}$ This study shows butorphanol as an effective pre-emptive analgesia with sevoflurane anesthesia. ${ }^{9}$ Exotic Animal Formulary 5 th edition dosing regimen recommendation of constant-rate infusion butorphanol in psittacine is $3 \mathrm{mg} / \mathrm{kg}$ (premedication) $+75 \mu \mathrm{g} / \mathrm{kg} / \mathrm{min}$ IV CRI (maintenance). ${ }^{10}$ Exotic Animal Formulary 5th edition dosing regimen recommendation of Intravenousbutorphanol in ratites is $0.05-0.25 \mathrm{mg} / \mathrm{kg}$. ${ }^{11}$ Infusion rate = target plasma butorphanol concentration $\times \mathrm{Cl}=60 \mu \mathrm{g} / \mathrm{L} \times 2 \mathrm{~L} / \mathrm{kg} / \mathrm{h}=120 \mu \mathrm{g} / \mathrm{kg} / \mathrm{h}$. Required concentration = infusion rate $\times$ bird's body weight $/$ temperature-adjusted pump rate $=120 \mu \mathrm{g} / \mathrm{kg} / \mathrm{h} \times 4.45 \mathrm{~kg} / 11.1 \mu \mathrm{L} / \mathrm{h}=48.1 \mu \mathrm{g} / \mu \mathrm{L}=48.1 \mathrm{mg} / \mathrm{mL}$ 12 Dosing regimen recommendation in Exotic Animal Formulary 5th edition is $0.1 \mathrm{mg} / \mathrm{kg}$, which is lower than dosage studied. ${ }^{13}$ Exotic Animal Formulary 5th edition dosing regimen recommendation of CRI fentanyl for Red-tailed Hawk is $20 \mu \mathrm{g}$ bolus $+0.2-0.5 \mu \mathrm{g} / \mathrm{kg} / \mathrm{min} .{ }^{14}$ Exotic Animal Formulary 5th edition dosing regimen recommendation of CRI fentanyl for Red-tailed Hawk is $20 \mu \mathrm{g}$ bolus $+1.5-6 \mu \mathrm{g} / \mathrm{kg} / \mathrm{min}$.

\section{Evaluation of Dosing Regimens in Relation to Efficacy Evidence}

The efficacy data for selected opioids administered to bird species studied are reflected in Table 3. PD studies selected were those which provided information on foot withdrawal thresholds to thermal or electrical stimuli after administration of selected opioids. A significant increase in foot withdrawal threshold to thermal or electrical stimuli after the administration of an opioid indicates the analgesic effect of the opioid used for the particular type of pain. PD studies may also utilize arthritis-induced birds. However, when PD studies were unavailable, PK studies were used to determine whether plasma concentrations of the selected opioids reached the target concentration. This target concentration is usually derived from plasma concentration of opioids associated with analgesia in humans with the exceptions of hydromorphone and butorphanol, where plasma concentrations associated with analgesia in American Kestrel and Hispaniolan Amazon were used, respectively. This plasma concentration was derived from PK and PD studies involving the administration of liposome-encapsulated butorphanol in Hispaniolan Amazon [50,52]. 
Table 3. Efficacy of selected opioids based on pharmacodynamics studies and plasma concentrations.

\begin{tabular}{|c|c|c|c|c|c|c|c|c|c|c|c|}
\hline \multicolumn{2}{|c|}{ Species Name } & \multicolumn{2}{|c|}{ Dosing Regimen } & \multicolumn{4}{|c|}{ PD Study } & \multicolumn{3}{|c|}{ Plasma Concentration } & \multirow[b]{2}{*}{ Reference } \\
\hline \multirow{2}{*}{ English } & \multirow{2}{*}{ Scientific } & \multirow{2}{*}{ Dose } & \multirow{2}{*}{ Administration } & \multirow{2}{*}{ Type } & \multirow{2}{*}{ Result } & \multirow{2}{*}{ Duration } & \multirow{2}{*}{ Target (Parent/Metabolite) } & \multirow{2}{*}{ Source } & \multicolumn{2}{|c|}{ Duration $\geq$ Target Concentration } & \\
\hline & & & & & & & & & Opioid & Metabolite & \\
\hline \multicolumn{12}{|c|}{ TRAMADOL } \\
\hline \multirow[t]{2}{*}{ Bald Eagle } & \multirow{2}{*}{$\begin{array}{l}\text { Haliaeetus } \\
\text { leucocephalus }\end{array}$} & $11 \mathrm{mg} / \mathrm{kg}$ & Oral & - & - & - & $298-590 \mathrm{ng} / \mathrm{mL} / 39.6-84 \mathrm{ng} / \mathrm{mL}$ & Human & $\begin{array}{c}10 \mathrm{~h} ; \\
5 / 6 \text { birds }\end{array}$ & $\begin{array}{c}2 \text { eagles; } \\
1 \text { timepoint each }\end{array}$ & \multirow[t]{2}{*}[24,25]{} \\
\hline & & $4 \mathrm{mg} / \mathrm{kg}$ & Intravenous & - & - & - & $298-590 \mathrm{ng} / \mathrm{mL} / 39.6-84 \mathrm{ng} / \mathrm{mL}$ & Human & $5 \mathrm{~h} ; 5 / 6$ birds & - & \\
\hline $\begin{array}{c}\text { American } \\
\text { Kestrel }\end{array}$ & $\begin{array}{c}\text { Falco } \\
\text { sparverius }\end{array}$ & $\begin{array}{l}5 \mathrm{mg} / \mathrm{kg} \\
15 \mathrm{mg} / \mathrm{kg} \\
30 \mathrm{mg} / \mathrm{kg}\end{array}$ & Oral & $\begin{array}{l}\text { Thermal } \\
\text { stimulus }\end{array}$ & $\begin{array}{l}\text { Significant increase in } \\
\text { thermal withdrawal } \\
\text { threshold. }\end{array}$ & $\begin{array}{c}9 \mathrm{~h} \text { for } \\
5 \mathrm{mg} / \mathrm{kg} ; 3 \mathrm{~h} \\
\text { for } 15 \mathrm{mg} / \mathrm{kg} \\
\text { and } \\
30 \mathrm{mg} / \mathrm{kg} .\end{array}$ & - & - & - & - & [26] \\
\hline $\begin{array}{l}\text { Red-tailed } \\
\text { Hawk }\end{array}$ & $\begin{array}{c}\text { Buteo } \\
\text { jamaicensis }\end{array}$ & $11 \mathrm{mg} / \mathrm{kg}$ & Oral & - & - & - & $298-590 \mathrm{ng} / \mathrm{mL} / 39.6-84 \mathrm{ng} / \mathrm{mL}$ & Human & $\begin{array}{l}4 \mathrm{~h} \text { after } \\
\text { dosing }\end{array}$ & - & {$[25,27]$} \\
\hline $\begin{array}{l}\text { Jackass } \\
\text { Penguin }\end{array}$ & $\begin{array}{l}\text { Spheniscus } \\
\text { Demersus }\end{array}$ & $10 \mathrm{mg} / \mathrm{kg}$ & Oral & - & - & - & $298-590 \mathrm{ng} / \mathrm{mL} / 39.6-84 \mathrm{ng} / \mathrm{mL}$ & Human & $\begin{array}{l}12 \mathrm{~h} \text { in } 9 / 15 \\
\text { of birds and } \\
24 \mathrm{~h} \text { in } 1 / 15 \\
\text { of birds }\end{array}$ & $\begin{array}{l}36 \mathrm{~h} \text { in } 14 / 15 \\
\text { of birds }\end{array}$ & [28] \\
\hline $\begin{array}{l}\text { Hispaniolan } \\
\text { Parrot }\end{array}$ & $\begin{array}{l}\text { Amazona } \\
\text { ventralis }\end{array}$ & $5 \mathrm{mg} / \mathrm{kg}$ & Intravenous & $\begin{array}{l}\text { Thermal } \\
\text { stimulus }\end{array}$ & $\begin{array}{l}\text { Increase in thermal } \\
\text { withdrawal threshold }\end{array}$ & & - & - & - & - & [29-32] \\
\hline $\begin{array}{l}\text { Indian } \\
\text { Peafowl }\end{array}$ & Pavo Cristatus & $7.5 \mathrm{mg} / \mathrm{kg}$ & Oral & - & - & - & $298-590 \mathrm{ng} / \mathrm{mL} / 39.6-84 \mathrm{ng} / \mathrm{mL}$ & Human & $\begin{array}{l}\text { Up to } 2 \mathrm{~h} \text { in } 2 \\
\text { birds }\end{array}$ & $\begin{array}{l}12 \mathrm{~h} \text { in } 5 / 6 \\
\text { birds }\end{array}$ & [33] \\
\hline $\begin{array}{l}\text { Muscovy } \\
\text { Duck }\end{array}$ & $\begin{array}{l}\text { Carina } \\
\text { moschata }\end{array}$ & $30 \mathrm{mg} / \mathrm{kg}$ & Oral & $\begin{array}{l}\text { Difference in } \\
\text { maximum } \\
\text { force } \\
\text { between } \\
\text { arthritis- } \\
\text { induced and } \\
\text { non-arthritis } \\
\text { induced legs }\end{array}$ & $\begin{array}{l}\text { Significant difference } \\
\text { between both } \\
\text { tramadol and } \\
\text { meloxicam vs. control }\end{array}$ & Up to $4 \mathrm{~h}$ & $298-590 \mathrm{ng} / \mathrm{mL} / 39.6-84 \mathrm{ng} / \mathrm{mL}$ & Human & At least $12 \mathrm{~h}$ & At least $6 \mathrm{~h}$ & {$[7,34]$} \\
\hline
\end{tabular}


Table 3. Cont.

\begin{tabular}{|c|c|c|c|c|c|c|c|c|c|c|c|}
\hline \multicolumn{2}{|c|}{ Species Name } & \multicolumn{2}{|c|}{ Dosing Regimen } & \multicolumn{4}{|c|}{ PD Study } & \multicolumn{3}{|c|}{ Plasma Concentration } & \\
\hline \multirow{2}{*}{ English } & \multirow{2}{*}{ Scientific } & \multirow{2}{*}{ Dose } & \multirow{2}{*}{ Administration } & \multirow{2}{*}{ Type } & \multirow{2}{*}{ Result } & \multirow{2}{*}{ Duration } & \multirow{2}{*}{ Target (Parent/Metabolite) } & \multirow{2}{*}{ Source } & \multicolumn{3}{|c|}{ Duration $\geq$ Target Concentration Reference } \\
\hline & & & & & & & & & Opioid & Metabolite & \\
\hline \multicolumn{12}{|c|}{ HYDROMORPHONE } \\
\hline $\begin{array}{c}\text { American } \\
\text { Kestrel }\end{array}$ & $\begin{array}{c}\text { Falco } \\
\text { sparverius }\end{array}$ & $\begin{array}{l}0.1 \mathrm{mg} / \mathrm{kg} \\
0.3 \mathrm{mg} / \mathrm{kg} \\
0.6 \mathrm{mg} / \mathrm{kg}\end{array}$ & Intramuscular & $\begin{array}{l}\text { Thermal } \\
\text { stimulus }\end{array}$ & $\begin{array}{c}0.6 \mathrm{mg} / \mathrm{kg}- \\
\text { Significant increase in } \\
\text { thermal foot } \\
\text { withdrawal threshold }\end{array}$ & $3 \mathrm{~h}$ & $>1 \mathrm{ng} / \mathrm{mL}$ & $\begin{array}{c}\text { American } \\
\text { kestrel }\end{array}$ & $\begin{array}{c}0.6 \mathrm{mg} / \mathrm{kg} \\
-3-6 \mathrm{~h}\end{array}$ & - & {$[35,36]$} \\
\hline Cockatiel & $\begin{array}{l}\text { Nymphicus } \\
\text { hollandicus }\end{array}$ & $\begin{array}{l}0.1 \mathrm{mg} / \mathrm{kg} \\
0.3 \mathrm{mg} / \mathrm{kg} \\
0.6 \mathrm{mg} / \mathrm{kg}\end{array}$ & Intramuscular & $\begin{array}{l}\text { Thermal } \\
\text { stimulus }\end{array}$ & $\begin{array}{c}\text { Did not increase } \\
\text { thermal foot } \\
\text { withdrawal threshold }\end{array}$ & - & $>1 \mathrm{ng} / \mathrm{mL}$ & $\begin{array}{c}\text { American } \\
\text { kestrel }\end{array}$ & $\begin{array}{c}0.6 \mathrm{mg} / \mathrm{kg}- \\
3-6 \mathrm{~h}\end{array}$ & - & {$[50]$} \\
\hline \multirow[t]{2}{*}{$\begin{array}{l}\text { Orange- } \\
\text { winged } \\
\text { Parrot }\end{array}$} & \multirow[t]{2}{*}{$\begin{array}{l}\text { Amazona } \\
\text { amazonica }\end{array}$} & $\begin{array}{l}0.1 \mathrm{mg} / \mathrm{kg} \\
1 \mathrm{mg} / \mathrm{kg} \\
2 \mathrm{mg} / \mathrm{kg}\end{array}$ & Intramuscular & $\begin{array}{l}\text { Thermal } \\
\text { stimulus }\end{array}$ & $\begin{array}{l}\text { Significant increase in } \\
\text { thermal foot } \\
\text { withdrawal threshold }\end{array}$ & $\begin{array}{c}\text { When } \\
\text { administered } \\
1 \mathrm{mg} / \mathrm{kg} \text { and } \\
2 \mathrm{mg} / \mathrm{kg}- \\
3 \mathrm{~h} \text { and } 6 \mathrm{~h} \\
\text { respectively }\end{array}$ & $>1 \mathrm{ng} / \mathrm{mL}$ & $\begin{array}{c}\text { American } \\
\text { kestrel }\end{array}$ & $\begin{array}{l}1 \mathrm{mg} / \mathrm{kg}- \\
6 \mathrm{~h} \text { in } 8 / 8 \text { birds }\end{array}$ & - & \multirow[t]{2}{*}[38,39]{} \\
\hline & & $1 \mathrm{mg} / \mathrm{kg}$ & Intravenous & -1 & - & - & $>1 \mathrm{ng} / \mathrm{mL}$ & $\begin{array}{c}\text { American } \\
\text { kestrel }\end{array}$ & $6 \mathrm{~h} ; 6 / 7$ birds & - & \\
\hline \multirow[t]{2}{*}{ Grey Parrot } & \multirow[t]{2}{*}{$\begin{array}{l}\text { Psittacus } \\
\text { erithacus }\end{array}$} & $0.1 \mathrm{mg} / \mathrm{kg}$ & Intramuscular & $\begin{array}{l}\text { Electric } \\
\text { stimulus }\end{array}$ & $\begin{array}{c}\text { No significant change } \\
\text { in electrical } \\
\text { withdrawal threshold }\end{array}$ & - & $>1 \mathrm{ng} / \mathrm{mL}$ & Human & $2 \mathrm{~h}$ & - & {$[40,41]$} \\
\hline & & $0.1 \mathrm{mg} / \mathrm{kg}$ & Intravenous & - & - & - & $>1 \mathrm{ng} / \mathrm{mL}$ & Human & $2 \mathrm{~h}$ & - & {$[40,41]$} \\
\hline \multirow{4}{*}{$\begin{array}{c}\text { American } \\
\text { Kestrel }\end{array}$} & \multirow{4}{*}{$\begin{array}{c}\text { Falco } \\
\text { sparverius }\end{array}$} & $\begin{array}{l}0.1 \mathrm{mg} / \mathrm{kg} \\
0.3 \mathrm{mg} / \mathrm{kg} \\
0.6 \mathrm{mg} / \mathrm{kg}\end{array}$ & Intramuscular & $\begin{array}{l}\text { Thermal } \\
\text { stimulus }\end{array}$ & $\begin{array}{l}\text { Significant increase in } \\
\text { thermal foot } \\
\text { withdrawal threshold }\end{array}$ & $\begin{array}{l}\text { All doses } \\
\text { tested-6h }\end{array}$ & $>1 \mathrm{ng} / \mathrm{mL}$ & Human & $0.6 \mathrm{mg} / \mathrm{kg}-9 \mathrm{~h}^{2}$ & - & {$[42,43]$} \\
\hline & & $1.8 \mathrm{mg} / \mathrm{kg}$ & $\begin{array}{l}\text { Subcutaneous } \\
\text { (sustained-release) }\end{array}$ & - & - & - & $>1 \mathrm{ng} / \mathrm{mL}$ & Human & $48 \mathrm{~h}$ & - & [44] \\
\hline & & $1.8 \mathrm{mg} / \mathrm{kg}$ & $\begin{array}{l}\text { Intramuscular } \\
\text { (sustained-release) }\end{array}$ & $\begin{array}{l}\text { Thermal } \\
\text { stimulus }\end{array}$ & $\begin{array}{l}\text { Significant increase in } \\
\text { thermal foot } \\
\text { withdrawal threshold }\end{array}$ & $\mathrm{Up}$ to $24 \mathrm{~h}$ & $>1 \mathrm{ng} / \mathrm{mL}$ & Humans & $48 \mathrm{~h}$ & - & {$[44,45]$} \\
\hline & & $0.6 \mathrm{mg} / \mathrm{kg}$ & Intravenous & - & - & - & $>1 \mathrm{ng} / \mathrm{mL}$ & Human & $9 \mathrm{~h}$ & - & [43] \\
\hline
\end{tabular}


Table 3. Cont.

\begin{tabular}{|c|c|c|c|c|c|c|c|c|c|c|c|}
\hline \multicolumn{2}{|c|}{ Species Name } & \multicolumn{2}{|c|}{ Dosing Regimen } & \multicolumn{4}{|c|}{ PD Study } & \multicolumn{3}{|c|}{ Plasma Concentration } & \multirow[b]{2}{*}{ Reference } \\
\hline \multirow{2}{*}{ English } & \multirow{2}{*}{ Scientific } & \multirow{2}{*}{ Dose } & \multirow{2}{*}{ Administration } & \multirow{2}{*}{ Type } & \multirow{2}{*}{ Result } & \multirow{2}{*}{ Duration } & \multirow{2}{*}{ Target (Parent/Metabolite) } & \multirow{2}{*}{ Source } & \multicolumn{2}{|c|}{ Duration $\geq$ Target Concentration } & \\
\hline & & & & & & & & & Opioid & Metabolite & \\
\hline \multirow[b]{2}{*}{$\begin{array}{l}\text { Red-tailed } \\
\text { Hawk }\end{array}$} & \multirow{2}{*}{$\begin{array}{c}\text { Buteo } \\
\text { jamaicensis }\end{array}$} & $0.25 \mathrm{mg} / \mathrm{kg}$ & Intramuscular & -3 & - & - & - & - & - & - & [46] \\
\hline & & $\begin{array}{l}0.3 \mathrm{mg} / \mathrm{kg} \\
1.8 \mathrm{mg} / \mathrm{kg}\end{array}$ & $\begin{array}{l}\text { Subcutaneous } \\
\text { (concentrated) }\end{array}$ & - & - & - & $>1 \mathrm{ng} / \mathrm{mL}$ & Human & $\begin{array}{l}0.3 \mathrm{mg} / \mathrm{kg}-24 \mathrm{~h} ; \\
1.8 \mathrm{mg} / \mathrm{kg}-48 \mathrm{~h}\end{array}$ & - & [47] \\
\hline Cockatiel & $\begin{array}{l}\text { Nymphicus } \\
\text { hollandicus }\end{array}$ & $\begin{array}{l}0.6 \mathrm{mg} / \mathrm{kg} \\
1.2 \mathrm{mg} / \mathrm{kg} \\
1.8 \mathrm{mg} / \mathrm{kg}\end{array}$ & Intramuscular & $\begin{array}{l}\text { Thermal } \\
\text { stimulus }\end{array}$ & $\begin{array}{c}\text { No significant } \\
\text { increase in thermal } \\
\text { withdrawal threshold }\end{array}$ & - & $>1 \mathrm{ng} / \mathrm{mL}$ & Human & $9 \mathrm{~h} ; 4 / 4$ birds & - & [48] \\
\hline \multicolumn{12}{|c|}{ BUTORPHANOL } \\
\hline \multirow{5}{*}{$\begin{array}{l}\text { Hispaniolan } \\
\text { Parrot }\end{array}$} & \multirow{5}{*}{$\begin{array}{l}\text { Amazona } \\
\text { ventralis }\end{array}$} & $5 \mathrm{mg} / \mathrm{kg}$ & Oral & - & - & - & $100 \mathrm{ng} / \mathrm{mL}$ & $\begin{array}{l}\text { Hispaniolan } \\
\text { amazon } \\
\text { parrots }\end{array}$ & 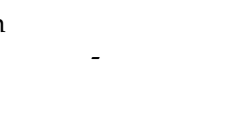 & - & [49] \\
\hline & & $\begin{array}{c}2 \mathrm{mg} / \mathrm{kg} 4 \\
5 \mathrm{mg} / \mathrm{kg}\end{array}$ & Intramuscular & - & - & - & $100 \mathrm{ng} / \mathrm{mL}$ & $\begin{array}{l}\text { Hispaniolan } \\
\text { amazon } \\
\text { parrots }\end{array}$ & - & - & {$[49,50]$} \\
\hline & & $5 \mathrm{mg} / \mathrm{kg}$ & Intravenous & - & - & - & $100 \mathrm{ng} / \mathrm{mL}$ & $\begin{array}{l}\text { Hispaniolan } \\
\text { amazon } \\
\text { parrots }\end{array}$ & 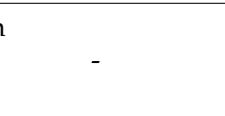 & - & [49] \\
\hline & & $\begin{array}{l}10 \mathrm{mg} / \mathrm{kg} \\
15 \mathrm{mg} / \mathrm{kg}\end{array}$ & $\begin{array}{l}\text { Subcutaneous } \\
\text { (Liposome- } \\
\text { Encapsulated) }\end{array}$ & $\begin{array}{l}\text { Electrical and } \\
\text { thermal } \\
\text { stimuli }\end{array}$ & $\begin{array}{l}\text { Significant increased } \\
\text { in electrical and } \\
\text { thermal withdrawal } \\
\text { threshold }\end{array}$ & Up to 5 days & -5 & - & - & - & {$[50,52]$} \\
\hline & & $12 \mathrm{mg} / \mathrm{kg}$ & $\begin{array}{l}\text { Subcutaneous } \\
\text { (Long-Acting) }\end{array}$ & - & - & - & $100 \mathrm{ng} / \mathrm{mL}$ & $\begin{array}{l}\text { Hispaniolan } \\
\text { amazon } \\
\text { parrots }\end{array}$ & $\begin{array}{c}>3 \mathrm{~h} \text { (all birds) } \\
\text { or }>4 \mathrm{~h} \\
(5 / 8 \text { birds }) \\
\text { but }<8 \mathrm{~h} \\
\text { (all birds) }\end{array}$ & - & [51] \\
\hline $\begin{array}{l}\text { Grey Parrot } \\
\text { and Timneh } \\
\text { Parrot }\end{array}$ & $\begin{array}{l}\text { Psittacus } \\
\text { erithacus and } \\
\text { Psittacus } \\
\text { timneh }\end{array}$ & $1 \mathrm{mg} / \mathrm{kg}$ & Intramuscular & $\begin{array}{l}\text { Electric } \\
\text { stimulus- }\end{array}$ & $\begin{array}{l}\text { Significant increase in } \\
\text { electrical withdrawal } \\
\text { threshold }\end{array}$ & - & - & - & - & - & [41] \\
\hline
\end{tabular}


Table 3. Cont.

\begin{tabular}{|c|c|c|c|c|c|c|c|c|c|c|c|}
\hline \multicolumn{2}{|c|}{ Species Name } & \multicolumn{2}{|c|}{ Dosing Regimen } & \multicolumn{4}{|c|}{ PD Study } & \multicolumn{3}{|c|}{ Plasma Concentration } & \multirow[b]{2}{*}{ Reference } \\
\hline \multirow{2}{*}{ English } & \multirow{2}{*}{ Scientific } & \multirow{2}{*}{ Dose } & \multirow{2}{*}{ Administration } & \multirow{2}{*}{ Type } & \multirow{2}{*}{ Result } & \multirow{2}{*}{ Duration } & \multirow{2}{*}{ Target (Parent/Metabolite) } & \multirow{2}{*}{ Source } & \multicolumn{2}{|c|}{ Duration $\geq$ Target Concentration } & \\
\hline & & & & & & & & & Opioid & Metabolite & \\
\hline $\begin{array}{l}\text { Sulphur- } \\
\text { crested } \\
\text { Cockatoo } \\
\text { and Yellow- } \\
\text { crested } \\
\text { Cockatoo }\end{array}$ & $\begin{array}{l}\text { Cacatua } \\
\text { galerita, } \\
\text { Cacatua } \\
\text { sulphurea cin- } \\
\text { trinocristata } \\
\text { and Cacatua } \\
\text { sulphurea } \\
\text { sulphurea }\end{array}$ & $1 \mathrm{mg} / \mathrm{kg}$ & Intramuscular & - & - & - & - & - & - & - & [16] \\
\hline \multirow{2}{*}{$\begin{array}{c}\text { Red-tailed } \\
\text { Hawk }\end{array}$} & \multirow{2}{*}{$\begin{array}{c}\text { Buteo } \\
\text { jamaicensis }\end{array}$} & $0.5 \mathrm{mg} / \mathrm{kg}$ & Intravenous & - & - & - & $9-30 \mathrm{ng} / \mathrm{mL}$ & $\begin{array}{c}\text { Dogs, } \\
\text { horses \& } \\
\text { llamas } 6\end{array}$ & $2-4 \mathrm{~h}$ & - & [53] \\
\hline & & $0.5 \mathrm{mg} / \mathrm{kg}$ & Intramuscular & - & - & - & $9-30 \mathrm{ng} / \mathrm{mL}$ & $\begin{array}{c}\text { Dogs, } \\
\text { horses \& } \\
\text { llamas } 7\end{array}$ & $2-4 \mathrm{~h}$ & - & [53] \\
\hline $\begin{array}{c}\text { Great } \\
\text { Horned Owl }\end{array}$ & $\begin{array}{c}\text { Bubo } \\
\text { virginianus }\end{array}$ & $0.5 \mathrm{mg} / \mathrm{kg}$ & Intravenous & - & - & - & $9-30 \mathrm{ng} / \mathrm{mL}$ & $\begin{array}{c}\text { Dogs, } \\
\text { horses \& } \\
\text { llamas } 8\end{array}$ & $2-4 \mathrm{~h}$ & - & [53] \\
\hline $\begin{array}{c}\text { American } \\
\text { Kestrel }\end{array}$ & $\begin{array}{c}\text { Falco } \\
\text { sparverius }\end{array}$ & $\begin{array}{l}1 \mathrm{mg} / \mathrm{kg} \\
3 \mathrm{mg} / \mathrm{kg} \\
6 \mathrm{mg} / \mathrm{kg}\end{array}$ & Intramuscular & $\begin{array}{l}\text { Thermal } \\
\text { stimulus }\end{array}$ & $\begin{array}{c}\text { Significantly } \\
\text { increased in female } \\
\text { compared with } \\
\text { baseline but not } \\
\text { significant compared } \\
\text { with control }\end{array}$ & - & $100 \mathrm{ng} / \mathrm{mL}$ & Humans & $2 \mathrm{~h}$ & - & [54] \\
\hline $\begin{array}{l}\text { Broiler } \\
\text { Chicken }\end{array}$ & - & $2 \mathrm{mg} / \mathrm{kg}$ & Intravenous & - & - & - & $55-70 \mathrm{ng} / \mathrm{mL}$ & Unknown & $2 \mathrm{~h}$ & - & [55] \\
\hline $\begin{array}{l}\text { Indian } \\
\text { Peafowl }\end{array}$ & Pavo cristatus & $\begin{array}{l}120 \mu \mathrm{g} / \mathrm{kg} / \mathrm{hr} \\
\text { with rate } \\
48.1 \mathrm{mg} / \mathrm{mL}^{10}\end{array}$ & $\begin{array}{l}\text { Osmotic } \\
\text { pump }\end{array}$ & - & - & - & $60 \mu \mathrm{g} / \mathrm{mL}$ & $\begin{array}{l}\text { Hispaniolan } \\
\text { amazon } \\
\text { parrots }\end{array}$ & 85.6 h (mean) & - & [56] \\
\hline
\end{tabular}


Table 3. Cont.

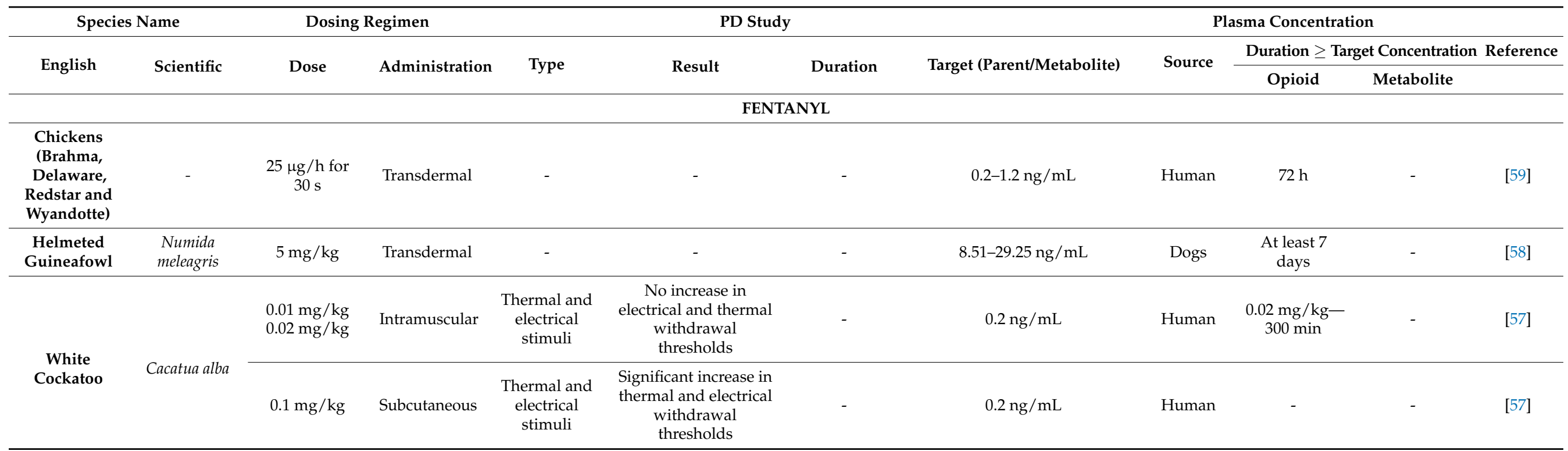

${ }^{1}$ Similar PK profile to that of IM hydromorphone. Efficacy can be assumed. ${ }^{2}$ PK data in birds treated with 0.1 and $0.3 \mathrm{mg} / \mathrm{kg}$ buprenorphine are not available. ${ }^{3}$ This study's main objective is not to evaluate antinociception nor study pharmacokinetics of buprenorphine but to evaluate behaviors associated with pain in red-tailed hawks. The study did not give direct evidence of analgesia after administration of buprenorphine to red-tailed hawks and buprenorphine did not return hawks with trauma to normal behavior. ${ }^{4}$ This study shows butorphanol as an effective pre-emptive analgesia with sevoflurane anesthesia. ${ }^{5}$ This study shows when butorphanol reach plasma concentration of $80 \mathrm{ng} / \mathrm{mL}$, withdrawal thresholds to electrical and thermal stimuli were increased. ${ }^{6}$ Based on study in dogs $(9-10 \mathrm{ng} / \mathrm{mL})$, horses $(20-30 \mathrm{ng} / \mathrm{mL})$ and $11 \mathrm{amas}(9.2-23.8 \mathrm{ng} / \mathrm{mL}){ }^{7}$ Based on study in dogs thresholds to electrical and thermal stimuli were increased. ${ }^{6}$ Based on study in dogs $(9-10 \mathrm{ng} / \mathrm{mL})$, horses $(20-30 \mathrm{ng} / \mathrm{mL})$ and llamas $(9.2-23.8 \mathrm{ng} / \mathrm{mL}) .{ }^{7}$ Based on study in dogs
$(9-10 \mathrm{ng} / \mathrm{mL})$, horses $(20-30 \mathrm{ng} / \mathrm{mL})$ and llamas $(9.2-23.8 \mathrm{ng} / \mathrm{mL}) .{ }^{8}$ Based on study in dogs $(9-10 \mathrm{ng} / \mathrm{mL})$, horses $(20-30 \mathrm{ng} / \mathrm{mL})$ and llamas $(9.2-23.8 \mathrm{ng} / \mathrm{mL}) .{ }^{9}$ Based on study $(9-10 \mathrm{ng} / \mathrm{mL})$, horses $(20-30 \mathrm{ng} / \mathrm{mL})$ and llamas $(9.2-23.8 \mathrm{ng} / \mathrm{mL}) .{ }^{8}$ Based on study in dogs $(9-10 \mathrm{ng} / \mathrm{mL})$, horses $(20-30 \mathrm{ng} / \mathrm{mL})$ and llamas $(9.2-23.8 \mathrm{ng} / \mathrm{mL}) .{ }^{9} \mathrm{Based} \mathrm{on} \mathrm{study}$
in dogs $(9-10 \mathrm{ng} / \mathrm{mL})$, horses $(20-30 \mathrm{ng} / \mathrm{mL})$ and llamas $(9.2-23.8 \mathrm{ng} / \mathrm{mL}) .1^{0}$ Infusion rate $=$ target plasma butorphanol concentration $\times \mathrm{Cl}=60 \mu \mathrm{gg} / \mathrm{L} \times 2 \mathrm{~L} / \mathrm{kg} / \mathrm{h}=120 \mathrm{\mu g} / \mathrm{kg} / \mathrm{h}$. Required concentration $=$ infusion rate $\times$ bird's body weight $/$ temperature-adjusted pump rate $=120 \mu \mathrm{g} / \mathrm{kg} / \mathrm{h} \times 4.45 \mathrm{~kg} / 11.1 \mu \mathrm{L} / \mathrm{h}=48.1 \mu \mathrm{g} / \mu \mathrm{L}=48.1 \mathrm{mg} / \mathrm{mL}$. 


\subsection{Tramadol}

The use of tramadol has been examined in several avian species including the Bald Eagle (Haliaetus leucocephalus) [24,25], American Kestrel (Falco sparverius) [26], Red-tailed Hawk (Buteo jamaicensis) [25,27], African Penguin (Spheniscus demersus) [28], Hispaniolan Amazon (Amazona ventralis) [29-32], Indian Peafowl (Pavo cristatus) [33] and Muscovy Duck (Carina moschata) [7,34]. Tramadol is a weak opioid agonist, which is highly specific to the $\mu$ receptor, which also acts as a weak serotonin and norepinephrine reuptake inhibitor [60-62]. Tramadol has numerous metabolites [63], but only O-desmethyltramadol (M1), which is a result of the demethylation of tramadol in the liver, has been shown to have clinically important analgesic properties in human [64].

The mean therapeutic plasma concentrations of tramadol and O-desmethyltramadol associated with analgesia in humans are $298 \pm 171 \mathrm{ng} / \mathrm{mL}$ to $590 \pm 410 \mathrm{ng} / \mathrm{mL}$ and $39.6 \pm 29.5 \mathrm{ng} / \mathrm{mL}$ to $84 \pm 34 \mathrm{ng} / \mathrm{mL}$, respectively $[65,66]$. Since the plasma therapeutic concentrations of tramadol and O-desmethyltramadol in avian species are unknown, efficacy is assumed if therapeutic plasma concentration of tramadol reached the plasma concentration associated with analgesia in human.

With this assumption, generally tramadol is effective in providing analgesia among the avian species studied. Target concentrations are reached among all species used in PK studies. The duration where plasma concentration reached or exceeded target concentration ranged from $2 \mathrm{~h}$ to at least $12 \mathrm{~h}$. However, only studies involving Muscovy Duck [7,34], American Kestrel [26] and Hispaniolan Amazon [29-31] are supported by PD evidences. These PD studies showed effectiveness of oral tramadol $30 \mathrm{mg} / \mathrm{kg}$, oral tramadol $5 \mathrm{mg} / \mathrm{kg}$ and oral tramadol $30 \mathrm{mg} / \mathrm{kg}$ in Muscovy Duck, American Kestrel and Hispaniolan Amazon, respectively, in providing an analgesic effect to thermal stimulus. In the study involving oral tramadol in American Kestrel, it is observed that a higher tramadol concentration i.e., $15 \mathrm{mg} / \mathrm{kg}$ and $30 \mathrm{mg} / \mathrm{kg}$ provided a shorter duration and increments in thermal threshold values of up to $3 \mathrm{~h}$ after administration compared with $9 \mathrm{~h}$ after administration of $5 \mathrm{mg} / \mathrm{kg}$ oral tramadol.

Separately, it was found in Hispaniolan Amazon that oral tramadol $10 \mathrm{mg} / \mathrm{kg}$ and $20 \mathrm{mg} / \mathrm{kg}$ did not significantly increase thermal foot withdrawal threshold, but oral tramadol $30 \mathrm{mg} / \mathrm{kg}$ significantly increased thermal foot withdrawal threshold for up to $6 \mathrm{~h}$. At the same time, a PK study also showed that $30 \mathrm{mg} / \mathrm{kg}$ oral tramadol given to Hispaniolan Amazon reached the minimum plasma concentration considered analgesic in humans for up to $6 \mathrm{~h}$. Since birds used for the 2 studies were from the same population, it can be assumed that results from the PK study can be applied to the PD study [31,32]. With that assumption, it could be deduced that the minimum effective plasma tramadol concentration of Hispaniolan Amazon is similar to humans.

Since birds do produce O-desmethyltramadol metabolites after the administration of tramadol, variability of analgesic effects of tramadol across different species may occur, depending on variability in biotransformation of tramadol in certain species [25]. However, given there is limited paired PK and PD studies to assess the efficacy of tramadol and there is no study assessing the analgesic effect of O-desmethyltramadol in birds, there is no consensus whether O-desmethyltramadol contributes to analgesic effect in avian species.

\subsection{Hydromorphone}

Hydromorphone, also known as dihydromorphinone, is a semi-synthetic $\mu$-opioid receptor agonist, which also displays weak affinity for $\mathrm{k}$-opioid receptors [67]. The use of hydromorphone has been examined in several avian species including American Kestrel (Falco sparverius) [35,36], Cockatiel (Nymphicus hollandicus) [37] and Orange-winged Amazon (Amazona amazonica) [38,39].

Since therapeutic plasma concentrations of hydromorphone is unknown in avian species, dosing recommendations were selected based on PK and PD studies done on American Kestrel, which suggested a thermal antinociceptive effect at plasma concentration of $>1 \mathrm{ng} / \mathrm{mL}[35,36]$. 
Generally, plasma concentrations of all species studied reached or exceeded plasma concentrations associated with analgesia in American Kestrel, with duration ranging from 3 to $6 \mathrm{~h}$. PD studies conducted in American Kestrel [35,36] and Orange-winged Amazon $[38,39]$ showed the effectiveness of hydromorphone in providing an analgesic effect to thermal stimuli at the given plasma concentration. One exception, however, is that of cockatiel [37]. Despite reaching plasma concentrations associated with analgesia in American kestrel, there is no significant increase in the thermal withdrawal threshold in cockatiel receiving $0.6 \mathrm{mg} / \mathrm{kg}$ hydromorphone [37]. The reason for this is unclear, but this highlights potential difference in analgesia mechanisms between cockatiel as compared to American Kestrel. Furthermore, opioid interactions with $\mu$-opioid receptors usually contributes to analgesic $[68,69]$ and adverse effects of opioids such as respiratory depression, constipation, sedation, nausea, vomiting, euphoria and withdrawal [70]. However, despite the presence of mild sedation for cockatiels receiving $0.3 \mathrm{mg} / \mathrm{kg}$ and $0.6 \mathrm{mg} / \mathrm{kg}$ IM hydromorphone, the thermal withdrawal threshold did not increase significantly [37]. This further emphasizes potential differences between the mechanisms that contribute to analgesic effects and adverse effects in cockatiels, as compared to other species of birds studied.

\subsection{Buprenorphine}

Buprenorphine is believed to be a mixed agonist/antagonist. It was reported that its analgesic action is largely from its $\mu$-opioid receptor agonism [71], but studies in rats and mice have shown buprenorphine antagonist action against $\mu$-opioid receptors [72]. Its action on k-opioid receptor also remains inconclusive [73-75]. Although the exact mechanism of its analgesic effect still remains uncertain [4], buprenorphine is shown to be an effective analgesic agent in animals [76].

Buprenorphine exhibits ceiling analgesic effect [77-80]. It binds strongly to opiate receptors, dissociates slowly from the receptors and it has a long-acting analgesic effect in mammalian species $[79,81]$. Plasma buprenorphine concentration may decline rapidly but its analgesic effect may remain, likely because of its strong binding to opiate receptors and slow dissociation from the receptors. Therefore, the relationship between plasma concentration and its analgesic effect may not be direct [79].

In humans, the plasma concentration of buprenorphine associated with analgesia is $>1-\mathrm{ng} / \mathrm{mL}$ [82]. Hence, the efficacy of buprenorphine in providing analgesic effect is generally assumed when the plasma concentration of buprenorphine in birds reaches $>1 \mathrm{ng} / \mathrm{mL}$.

Use of buprenorphine in avian species have been examined in Grey Parrot (Psittacus errithacus) [40,41], American Kestrel (Falco sparverius) [42,43], Red-tailed hawk (Buteo jamaicensis) [46], Rock Dove (Columba livia) [83] and Cockatiel (Nymphicus hollandicus) [48].

Generally, plasma concentrations of buprenorphine in bird species studied reached or exceeded buprenorphine plasma concentration associated with analgesia in human $(1 \mathrm{ng} / \mathrm{mL})$. PD studies done also showed an increase in thermal withdrawal threshold in American Kestrel treated with buprenorphine. However, a separate PD study involving Grey Parrot [40,41] and Cockatiel [48] showed no significant increase in electrical and thermal withdrawal thresholds at the doses given. A PK study performed on cockatiels did not observe analgesic effect even though the plasma concentration of buprenorphine reached target concentration for $9 \mathrm{~h}$.

In addition, the mean withdrawal threshold in male American Kestrel was significantly higher than female American Kestrel, highlighting a potential difference attributable to $\operatorname{sex}[42,43]$.

\subsection{Butorphanol}

Butorphanol is a synthetic, mixed agonist/antagonist at the $\mu$-opioid receptors. It is also a partial agonist at the $\mathrm{k}$-opioid receptors, which is present at a higher proportion in birds as compared to other species [84]. The affinity of butorphanol is reported to be stronger to $\mathrm{K}$-opioid receptors as compared to $\mu$-opioid receptors $[85,86]$. 
The target plasma concentration associated with analgesia in articles collated are different for each study. These target concentrations are reflected in Table 3.

Butorphanol has been studied in Grey Parrot and Timneh Parrot (Psittacus erithacus and Psittacus timneh) [41], Red-tailed Hawk (Buteo jamaicensis) [53], Great Horned Owl (Bubo virginianus) [53], Hispaniolan Amazon (Amazona ventralis) [49-51], Broiler Chicken [55], Indian Peafowl (Pavo cristatus) [56], Sulphur-crested Cockatoo and Yellow-crested Cockatoo (Cacatua galerita, Cacatua sulphurea cintrinocristata and Cacatua sulphurea sulphurea) [16] and American Kestrel (Falco sparverius) [54].

Generally, the use of butorphanol is likely to be effective in all species studied as various PK studies saw the plasma concentration of butorphanol reaching or exceeding target plasma concentration in the species studied. PD studies had also shown significant increase in thermal or electric withdrawal stimuli, except for male American Kestrel. Interestingly, hyperalgesia or hyperesthesia were observed at $1.5 \mathrm{~h}(\mathrm{Cmax})$ in male Kestrels administered $6 \mathrm{mg} / \mathrm{kg}$ butorphanol [54]. This is a sex dependent response between male and female American Kestrel, whereby thermal withdrawal thresholds, when compared with baseline value, were significantly decreased in male compared to significantly increased in female. The reason for this is unclear.

\subsection{Fentanyl}

Fentanyl is a synthetic, short-acting opiate, which produces its analgesic effect through $\mu$-opioid receptor agonism [87]. The use of fentanyl have been studied in Helmeted Guineafowl (Numida meleagris) [58], chicken [44,45] and White Cockatoo (Cacatua alba) [57].

Generally, the plasma concentration of all species studied reached or exceeded target concentration. PD studies to evaluate the effectiveness of fentanyl in producing analgesic effect to thermal and electrical stimuli were studied only in White cockatoo. Despite plasma concentration of White Cockatoo reaching plasma concentration associated with analgesia in humans when administered $0.01 \mathrm{mg} / \mathrm{kg}$ and $0.02 \mathrm{mg} / \mathrm{kg}$ of fentanyl, there was no significant increase in withdrawal threshold to electrical and thermal stimuli. However, significant increase in thermal and electrical withdrawal threshold were noticed when 10fold increase in dose $(0.2 \mathrm{mg} / \mathrm{kg})$ were administered. It appears that White Cockatoo might need higher plasma concentrations of fentanyl to provide analgesia, as compared to human, which further emphasize differences between species that may lead to different outcomes.

\subsection{Special Formulations of Opioids Impacting Efficacy of Opioids}

Other than standard formulations, special formulations of buprenorphine and butorphanol were also studied in avian species. Concentrated and sustained-release formulation of buprenorphine were studied in Red-tailed Hawk [47] and American Kestrel [44,45], respectively. Long-acting (LA) poloxamer 407 gel formulation [51] butorphanol and liposomeencapsulated butorphanol (LEBT) [50,52] were studied in Hispaniolan Amazon.

\subsubsection{Buprenorphine}

Both the concentrated formulation of buprenorphine and sustained-release buprenorphine provided longer duration where plasma concentrations of buprenorphine were at or above the target concentration, implying longer duration of effectiveness in providing analgesic effect. In red-tailed hawk, administration of $0.3 \mathrm{mg} / \mathrm{kg}$ and $1.8 \mathrm{mg} / \mathrm{kg}$ concentrated buprenorphine resulted in a duration where plasma concentration of buprenorphine reached or exceeded target concentration for $24 \mathrm{~h}$ and $48 \mathrm{~h}$, respectively. In addition, administration of $1.8 \mathrm{mg} / \mathrm{kg}$ sustained-release buprenorphine SC and IM to American Kestrel resulted in duration where plasma concentration of buprenorphine reached or exceeded target concentration for $48 \mathrm{~h}$. These durations are longer than that of standard formulation studied in other bird species, whereby duration where plasma concentration reached or exceeded target concentration ranged from 2-9 $\mathrm{h}$. Therefore, these formulations of buprenorphine could be used clinically if longer duration of action is desired. 


\subsubsection{Butorphanol}

Liposome-encapsulated butorphanol was studied in Hispaniolan Amazon. However, the duration where plasma concentration of butorphanol was at or beyond target concentration was not reported and hence its ability to provide longer analgesic effect is still unclear. On the other hand, LA butorphanol produced plasma concentrations at or beyond target concentration for up to $4-8 \mathrm{~h}$, which is longer than that of other formulation studied, where range of duration were typically only up to $4 \mathrm{~h}$. Hence, LA butorphanol could be used clinically, if deemed necessary.

\section{Trends in Efficacy of Selected Opioids}

Generally, at the doses given, plasma concentration of opioids among most bird species reached or exceeded target concentration. Furthermore, analgesic effects were observed among species studied.

Interestingly, Cockatiel did not seem to benefit from analgesic effect of $\mu$-receptor agonists, hydromorphone and buprenorphine, despite reaching target plasma concentration. The reason for this is unclear. However, a study comparing expressions of opioid receptors between Cockatiel and Rock Dove showed that Cockatiel has less $\mu$-opioid receptor expressions in the footpad as compared to Rock Dove [88]. This may explain the reason for non-significant increase in thermal or electrical withdrawal threshold in cockatiels despite presence of adverse effects associated with interactions with $\mu$-receptor. However, further studies need to be done to assess this.

Another observation point is the sex-dependent response between male and female American Kestrel after administration of buprenorphine and butorphanol. Although the exact reason is unclear, it is noted that American Kestrel exhibit sexual dimorphism, whereby female American Kestrel are generally larger and heavier than male Amarican Kestrel [89]. Weight may affect PK in terms of absorption or distribution, which may explain difference in responses between male and female American Kestrel. However, this may not be true as the use of tramadol and hydromorphone in American Kestrel did not produce significant sex-dependent response. Further studies need to be conducted to investigate the sex-dependent response.

Special formulations of buprenorphine and butorphanol were studied and resulted in longer duration where plasma concentration was reached or exceeded target concentration, implying longer duration of analgesic effect in the species studied. These formulations could be used clinically if longer duration of analgesic effect is deemed necessary.

\section{Evaluation of Dosing Regimens in Relation to Safety Evidence}

Table 4 shows the adverse effects of selected opioids used in particular species of birds. The use of opioids appeared to be safe for avian species, with the most common adverse effect being sedation. There was no severe adverse event such as death observed with the use of these opioids, although there were a few moderate adverse events such as ataxia, apnea, miosis, vomiting and bradycardia that was not clinically significant. It was also observed, from studies administering more than 1 dosing regimen of a specific opioids to a particular species of birds, that adverse events were generally dose-dependent. Gastrointestinal (GI) adverse effects of tramadol were observed in American Kestrels only when they were administered $15 \mathrm{mg} / \mathrm{kg}$ and $30 \mathrm{mg} / \mathrm{kg}$ tramadol, but this adverse effect was not observed when kestrels were given $5 \mathrm{mg} / \mathrm{kg}$ tramadol. 
Table 4. Adverse effects of selected opioids reported in species studied.

\begin{tabular}{|c|c|c|c|c|c|}
\hline \multicolumn{2}{|c|}{ Species Name } & \multicolumn{2}{|c|}{ Dosing Regimen } & \multirow[t]{2}{*}{ Adverse Effect } & \multirow[t]{2}{*}{ Reference } \\
\hline English & Scientific & Dose & Administration & & \\
\hline \multicolumn{6}{|c|}{ TRAMADOL } \\
\hline \multirow{2}{*}{ Bald Eagle } & \multirow{2}{*}{$\begin{array}{l}\text { Haliaeetus } \\
\text { leucocephalus }\end{array}$} & $11 \mathrm{mg} / \mathrm{kg}$ & Oral & None observed & \multirow{2}{*}[24,25]{} \\
\hline & & $4 \mathrm{mg} / \mathrm{kg}$ & Intravenous & $\begin{array}{l}\text { Mild transient bradycardia observed } \\
\text { immediately after administration until } 10 \text { min } \\
\text { after IV administration in } 3 \text { birds. Bradycardia } \\
\text { is deemed clinically insignificant. }\end{array}$ & \\
\hline $\begin{array}{l}\text { American } \\
\text { Kestrel }\end{array}$ & Falco sparverius & $\begin{array}{l}5 \mathrm{mg} / \mathrm{kg} \\
15 \mathrm{mg} / \mathrm{kg} \\
30 \mathrm{mg} / \mathrm{kg}\end{array}$ & Oral & $\begin{array}{l}\text { GI adverse effect observed at higher doses (15 } \\
\mathrm{mg} / \mathrm{kg} \text { and } 30 \mathrm{mg} / \mathrm{kg} \text { ). Vomiting observed in } 1 \\
\mathrm{kestrel} \mathrm{within} 30 \mathrm{~min} \text { after administration of } \\
\text { tramadol } 30 \mathrm{mg} / \mathrm{kg} \text {. Polyuria observed for } 6 \\
\text { birds- } 1 \text { control; } 2 \text { treated with } 15 \mathrm{mg} / \mathrm{kg} \\
\text { tramadol; } 3 \text { treated with } 30 \mathrm{mg} / \mathrm{kg}\end{array}$ & [26] \\
\hline $\begin{array}{l}\text { Red-tailed } \\
\text { Hawk }\end{array}$ & $\begin{array}{c}\text { Buteo } \\
\text { jamaicensis }\end{array}$ & $11 \mathrm{mg} / \mathrm{kg}$ & Oral & None observed & {$[25,27]$} \\
\hline $\begin{array}{l}\text { Jackass } \\
\text { Penguin }\end{array}$ & $\begin{array}{l}\text { Spheniscus } \\
\text { Demersus }\end{array}$ & $10 \mathrm{mg} / \mathrm{kg}$ & Oral & $\begin{array}{l}\text { Penguins were subjectively quieter } 1-1.5 \mathrm{~h} \text { post } \\
\text { administration \& considered back to normal } \\
\text { after } 6 \mathrm{~h} \text { of administration. This could be due to } \\
\text { frequent handling. Monitor for signs of } \\
\text { sedation. }\end{array}$ & {$[28]$} \\
\hline \multirow[t]{2}{*}{$\begin{array}{l}\text { Hispaniolan } \\
\text { Parrot }\end{array}$} & \multirow[t]{2}{*}{$\begin{array}{l}\text { Amazona } \\
\text { ventralis }\end{array}$} & $30 \mathrm{mg} / \mathrm{kg}$ & Oral & \multirow{2}{*}{$\begin{array}{l}\text { No sedation or changes in behavior were } \\
\text { detected. Birds had ruffled feathers and were } \\
\text { quiet after handling but did not have other } \\
\text { signs of stress. }\end{array}$} & [29-32] \\
\hline & & $5 \mathrm{mg} / \mathrm{kg}$ & Intravenous & & [29-32] \\
\hline $\begin{array}{l}\text { Indian } \\
\text { Peafowl }\end{array}$ & Pavo Cristatus & $7.5 \mathrm{mg} / \mathrm{kg}$ & Oral & No adverse effects or changes in behavior noted & [33] \\
\hline $\begin{array}{l}\text { Muscovy } \\
\text { Duck }\end{array}$ & Carina moschata & $30 \mathrm{mg} / \mathrm{kg}$ & Oral & No adverse effects noted & {$[7,34]$} \\
\hline \multicolumn{6}{|c|}{ HYDROMORPHONE } \\
\hline $\begin{array}{l}\text { American } \\
\text { Kestrel }\end{array}$ & Falco sparverius & $\begin{array}{l}0.1 \mathrm{mg} / \mathrm{kg} \\
0.3 \mathrm{mg} / \mathrm{kg} \\
0.6 \mathrm{mg} / \mathrm{kg}\end{array}$ & Intramuscular & $\begin{array}{l}\text { Appreciable sedation detected in } 4 \text { birds after } \\
\text { administration of } 6 \mathrm{mg} / \mathrm{kg} \text { hydromorphone }\end{array}$ & {$[35,36]$} \\
\hline Cockatiel & $\begin{array}{l}\text { Nymphicus } \\
\text { hollandicus }\end{array}$ & $\begin{array}{l}0.1 \mathrm{mg} / \mathrm{kg} \\
0.3 \mathrm{mg} / \mathrm{kg} \\
0.6 \mathrm{mg} / \mathrm{kg}\end{array}$ & Intramuscular & $\begin{array}{l}\text { Sedation after administration of } 0.3 \mathrm{mg} / \mathrm{kg} \text { and } \\
0.6 \mathrm{mg} / \mathrm{kg} \text { hydromorphone }\end{array}$ & {$[50]$} \\
\hline \multirow{2}{*}{$\begin{array}{l}\text { Orange- } \\
\text { winged } \\
\text { Parrot }\end{array}$} & \multirow[t]{2}{*}{$\begin{array}{l}\text { Amazona } \\
\text { amazonica }\end{array}$} & $\begin{array}{l}0.1 \mathrm{mg} / \mathrm{kg} \\
1 \mathrm{mg} / \mathrm{kg} \\
2 \mathrm{mg} / \mathrm{kg}\end{array}$ & Intramuscular & $\begin{array}{l}\text { Treatment was significantly }(p<0.001) \\
\text { associated with nausea-like behavior. Other } \\
\text { adverse effect includes ataxia and miosis. }\end{array}$ & \multirow[t]{2}{*}[38,39]{} \\
\hline & & $1 \mathrm{mg} / \mathrm{kg}$ & Intravenous & Not reported & \\
\hline \multicolumn{6}{|c|}{ BUPRENORPHINE } \\
\hline \multirow{2}{*}{ Grey Parrot } & \multirow{2}{*}{$\begin{array}{l}\text { Psittacus } \\
\text { erithacus }\end{array}$} & $0.1 \mathrm{mg} / \mathrm{kg}$ & Intramuscular & \multirow{2}{*}{ Not reported } & \multirow{2}{*}[40,41]{} \\
\hline & & $0.1 \mathrm{mg} / \mathrm{kg}$ & Intravenous & & \\
\hline
\end{tabular}


Table 4. Cont.

\begin{tabular}{|c|c|c|c|c|c|}
\hline \multicolumn{2}{|c|}{ Species Name } & \multicolumn{2}{|c|}{ Dosing Regimen } & \multirow[t]{2}{*}{ Adverse Effect } & \multirow[t]{2}{*}{ Reference } \\
\hline English & Scientific & Dose & Administration & & \\
\hline \multirow{4}{*}{$\begin{array}{l}\text { American } \\
\text { Kestrel }\end{array}$} & \multirow{4}{*}{ Falco sparverius } & $\begin{array}{l}0.1 \mathrm{mg} / \mathrm{kg} \\
0.3 \mathrm{mg} / \mathrm{kg} \\
0.6 \mathrm{mg} / \mathrm{kg}\end{array}$ & Intramuscular & \multirow{2}{*}{$\begin{array}{l}\text { Mild to moderate sedation was recorded within } \\
15 \text { to } 30 \text { min after both IM and IV } \\
\text { administration of } 0.6 \mathrm{mg} \text { of buprenorphine } / \mathrm{kg} \text {, } \\
\text { although the birds remained reactive to } \\
\text { physical handling at all time points. }\end{array}$} & {$[42,43]$} \\
\hline & & $0.6 \mathrm{mg} / \mathrm{kg}$ & Intravenous & & [43] \\
\hline & & $1.8 \mathrm{mg} / \mathrm{kg}$ & $\begin{array}{l}\text { Subcutaneous } \\
\text { (sustained-release) }\end{array}$ & \multirow{2}{*}{$\begin{array}{l}\text { There was no subjectively } \\
\text { appreciable sedation after } \\
\text { either IM or SC administration.- }\end{array}$} & {$[44]$} \\
\hline & & $1.8 \mathrm{mg} / \mathrm{kg}$ & $\begin{array}{l}\text { Intramuscular } \\
\text { (sustained-release) }\end{array}$ & & {$[44,45]$} \\
\hline & & $0.25 \mathrm{mg} / \mathrm{kg}$ & Intramuscular & -1 & [46] \\
\hline $\begin{array}{l}\text { Red-tailed } \\
\text { Hawk }\end{array}$ & $\begin{array}{c}\text { Buteo } \\
\text { jamaicensis }\end{array}$ & $\begin{array}{l}0.3 \mathrm{mg} / \mathrm{kg} \\
1.8 \mathrm{mg} / \mathrm{kg}\end{array}$ & $\begin{array}{l}\text { Subcutaneous } \\
\text { (concentrated) }\end{array}$ & $\begin{array}{c}\text { Mean agitation-sedation scores were higher } \\
\text { (indicating some degree of sedation) than the } \\
\text { baseline values for } 24 \mathrm{~h} \text { at both doses. No } \\
\text { clinically important adverse effects were } \\
\text { observed. }\end{array}$ & [47] \\
\hline Cockatiel & $\begin{array}{l}\text { Nymphicus } \\
\text { hollandicus }\end{array}$ & $\begin{array}{l}0.6 \mathrm{mg} / \mathrm{kg} \\
1.2 \mathrm{mg} / \mathrm{kg} \\
1.8 \mathrm{mg} / \mathrm{kg}\end{array}$ & Intramuscular & $\begin{array}{c}\text { Did not significantly cause sedative or } \\
\text { agitative effects }\end{array}$ & [48] \\
\hline \multicolumn{6}{|c|}{ BUTORPHANOL } \\
\hline \multirow{5}{*}{$\begin{array}{l}\text { Hispaniolan } \\
\quad \text { Parrot }\end{array}$} & \multirow{5}{*}{$\begin{array}{l}\text { Amazona } \\
\text { ventralis }\end{array}$} & $5 \mathrm{mg} / \mathrm{kg}$ & Oral & Significant adverse effects were not observed & [49] \\
\hline & & $\begin{array}{c}2 \mathrm{mg} / \mathrm{kg}^{2} \\
5 \mathrm{mg} / \mathrm{kg}\end{array}$ & Intramuscular & Significant adverse effects were not observed & {$[49,50]$} \\
\hline & & $5 \mathrm{mg} / \mathrm{kg}$ & Intravenous & $\begin{array}{l}\text { Significant adverse effects were not observed } \\
\text { but } 1 \text { parrot developed apnea for a short period. }\end{array}$ & [49] \\
\hline & & $\begin{array}{l}10 \mathrm{mg} / \mathrm{kg} \\
15 \mathrm{mg} / \mathrm{kg}\end{array}$ & $\begin{array}{l}\text { Subcutaneous } \\
\text { (Liposome- } \\
\text { Encapsulated) }\end{array}$ & $\begin{array}{l}\text { No observable adverse effect although there } \\
\text { were mild-moderate sedation between } 1-2 \mathrm{~h} \\
\text { after administration. }\end{array}$ & {$[50,52]$} \\
\hline & & $12 \mathrm{mg} / \mathrm{kg}$ & $\begin{array}{l}\text { Subcutaneous } \\
\text { (Long-Acting) }\end{array}$ & $\begin{array}{l}\text { All birds were subjectively quieter after } \\
\text { administration of butorphanol. Furthermore, } \\
2 \text { birds receiving } 16.6 \mathrm{mg} / \mathrm{kg} \text { SC vomited } \\
\text { around } 1.31 \mathrm{~h} \text { post administration. }\end{array}$ & [51] \\
\hline $\begin{array}{l}\text { Grey Parrot } \\
\text { and Timneh } \\
\text { Parrot }\end{array}$ & $\begin{array}{c}\text { Psittacus } \\
\text { erithacus and } \\
\text { Psittacus timneh }\end{array}$ & $1 \mathrm{mg} / \mathrm{kg}$ & Intramuscular & Not reported & [41] \\
\hline $\begin{array}{l}\text { Sulphur- } \\
\text { crested } \\
\text { Cockatoo and } \\
\text { Yellow-crested } \\
\text { Cockatoo }\end{array}$ & $\begin{array}{c}\text { Cacatua galerita, } \\
\text { Cacatua } \\
\text { sulphurea } \\
\text { cintrinocristata } \\
\text { and Cacatua } \\
\text { sulphurea } \\
\text { sulphurea }\end{array}$ & $1 \mathrm{mg} / \mathrm{kg}$ & Intramuscular & Not reported & [16] \\
\hline \multirow{2}{*}{$\begin{array}{l}\text { Red-tailed } \\
\text { Hawk }\end{array}$} & \multirow{2}{*}{$\begin{array}{c}\text { Buteo } \\
\text { jamaicensis }\end{array}$} & $0.5 \mathrm{mg} / \mathrm{kg}$ & Intravenous & \multirow{2}{*}{$\begin{array}{l}\text { Mild sedation but no significant change in heart } \\
\text { and respiratory rates }\end{array}$} & [53] \\
\hline & & $0.5 \mathrm{mg} / \mathrm{kg}$ & Intramuscular & & [53] \\
\hline \multirow{2}{*}{$\begin{array}{l}\text { Great Horned } \\
\text { Owl }\end{array}$} & \multirow{2}{*}{$\begin{array}{l}\text { Bubo } \\
\text { virginianus }\end{array}$} & $0.5 \mathrm{mg} / \mathrm{kg}$ & Intravenous & \multirow{2}{*}{$\begin{array}{l}\text { Mild sedation but no significant change in heart } \\
\text { and respiratory rates }\end{array}$} & [53] \\
\hline & & $0.5 \mathrm{mg} / \mathrm{kg}$ & Intramuscular & & [53] \\
\hline
\end{tabular}


Table 4. Cont.

\begin{tabular}{ccccc}
\hline \multicolumn{2}{c}{ Species Name } & \multicolumn{2}{c}{ Dosing Regimen } & Adverse Effect \\
\hline English & Scientific & Dose & Administration & \\
\hline $\begin{array}{c}\text { American } \\
\text { Kestrel }\end{array}$ & Falco sparverius & $\begin{array}{c}1 \mathrm{mg} / \mathrm{kg} \\
3 \mathrm{mg} / \mathrm{kg} \\
6 \mathrm{mg} / \mathrm{kg}\end{array}$ & Intramuscular & $\begin{array}{c}\text { No sedative effect observed but male kestrels } \\
\text { appeared agitated at 1.5 h. }\end{array}$ \\
\hline $\begin{array}{c}\text { Broiler } \\
\text { Chicken }\end{array}$ & - & $2 \mathrm{mg} / \mathrm{kg}$ & Intravenous & Not reported \\
\hline $\begin{array}{c}\text { Indian } \\
\text { Peafowl }\end{array}$ & Pavo cristatus & $\begin{array}{c}120 \mu \mathrm{mg} / \mathrm{kg} / \mathrm{h} \\
\text { with rate } \\
48.1 \mathrm{mg} / \mathrm{mL}^{3}\end{array}$ & $\begin{array}{c}\text { Osmotic } \\
\text { pump }\end{array}$ & No evidence of sedation or other adverse effect \\
\hline
\end{tabular}

FENTANYL

\begin{tabular}{|c|c|c|c|c|c|}
\hline $\begin{array}{c}\text { Chickens } \\
\text { (Brahma, } \\
\text { Delaware, } \\
\text { Redstar and } \\
\text { Wyandotte) }\end{array}$ & - & $\begin{array}{c}25 \mu \mathrm{g} / \mathrm{h} \text { for } \\
30 \mathrm{~s}\end{array}$ & Transdermal & No adverse effects noted & [59] \\
\hline $\begin{array}{l}\text { Helmeted } \\
\text { Guineafowl }\end{array}$ & $\begin{array}{l}\text { Numida } \\
\text { meleagris }\end{array}$ & $5 \mathrm{mg} / \mathrm{kg}$ & Transdermal & No adverse effects noted & [58] \\
\hline \multirow{2}{*}{$\begin{array}{l}\text { White } \\
\text { Cockatoo }\end{array}$} & \multirow{2}{*}{ Cacatua alba } & $\begin{array}{l}0.01 \mathrm{mg} / \mathrm{kg} \\
0.02 \mathrm{mg} / \mathrm{kg}\end{array}$ & Intramuscular & None observed & [57] \\
\hline & & $0.1 \mathrm{mg} / \mathrm{kg}$ & Subcutaneous & $\begin{array}{c}2 / 7 \text { birds sedate at } 20 \mathrm{~min}, 6 \text { birds hyperactive } \\
\text { between } 20 \text { and } 60 \mathrm{~min}\end{array}$ & [57] \\
\hline
\end{tabular}

1 This study's main objective is not to evaluate antinociception nor study pharmacokinetics of buprenorphine but to evaluate behaviors associated with pain in red-tailed hawks. The study did not give direct evidence of analgesia after administration of buprenorphine to Red-tailed Hawks and buprenorphine did not return hawks with trauma to normal behavior. 2 This study shows butorphanol as an effective pre-emptive analgesia with sevoflurane anesthesia. ${ }^{3}$ Infusion rate $=$ target plasma butorphanol concentration $\times \mathrm{Cl}=60 \mu \mathrm{g} / \mathrm{L} \times 2 \mathrm{~L} / \mathrm{kg} / \mathrm{h}=120 \mu \mathrm{g} / \mathrm{kg} / \mathrm{h}$ Required concentration $=$ infusion rate $\times$ bird's body weight/temperature-adjusted pump rate $=120 \mu \mathrm{g} / \mathrm{kg} / \mathrm{h} \times 4.45 \mathrm{~kg} / 11.1 \mu \mathrm{L} / \mathrm{h}=48.1 \mu \mathrm{g} / \mu \mathrm{L}=48.1 \mathrm{mg} / \mathrm{mL}$.

Another observation was the sex-dependent adverse effect in American Kestrels after the administration of butorphanol [54]. In this case, sedative effect was not observed but male Kestrels seemed to be agitated at $1.5 \mathrm{~h}$ post-administration. This observation was aligned with the observed sex-dependent response of efficacy in American Kestrels administered butorphanol.

\section{Pharmacokinetics Variability}

Table 5 shows the reported half-lives of selected opioids and their metabolites (if applicable) in various avian species studied. 
Table 5. The reported half-lives of selected opioids in avian species studied.

\begin{tabular}{|c|c|c|c|c|c|c|c|c|c|c|c|}
\hline \multicolumn{2}{|c|}{ Species } & \multirow{2}{*}{ Weight } & \multirow{2}{*}{ Dosage Studied } & \multirow{2}{*}{$\begin{array}{c}\text { Route of } \\
\text { Administration }\end{array}$} & \multirow{2}{*}{ Fed or Fasted } & \multirow{2}{*}{ Formulation } & \multirow{2}{*}{$\mathrm{C}_{\max }(\mathrm{ng} / \mathrm{mL})$} & \multirow{2}{*}{$\begin{array}{l}\text { Bioavailability } \\
(\%)\end{array}$} & \multicolumn{2}{|c|}{ Half-Life (hr) } & \multirow{2}{*}{ Reference } \\
\hline English & Scientific & & & & & & & & Parent Drug & Metabolite & \\
\hline \multicolumn{12}{|c|}{ TRAMADOL } \\
\hline Bald Eagle & $\begin{array}{c}\text { Haliaeetus } \\
\text { leucocephalus }\end{array}$ & $2.95-5.91 \mathrm{~kg}$ & $11 \mathrm{mg} / \mathrm{kg}$ & Oral & Fasted $12 \mathrm{~h}$ before dosing & Suspension & $2156.7 \pm 681.4$ & 97.9 & $3.14 \pm 0.94$ & $6.28 \pm 1.72$ & {$[24,25]$} \\
\hline Red-tailed Hawk & Buteo jamaicensis & Not reported & $11 \mathrm{mg} / \mathrm{kg}$ & Oral & Fasted $12 \mathrm{~h}$ before dosing & Suspension & $1412.3 \pm 79.6$ & - & $1.3 \pm 0.6$ & $1.9 \pm 0.2$ & {$[25,27]$} \\
\hline Jackass Penguin & $\begin{array}{l}\text { Spheniscus } \\
\text { Demersus }\end{array}$ & $\geq 2.7 \mathrm{~kg}$ & $10 \mathrm{mg} / \mathrm{kg}$ & Oral & $\begin{array}{l}\text { Tramadol given with a fish. No } \\
\text { additional fish offered untl } 4 \mathrm{~h} \text { later. }\end{array}$ & Capsules & - & - & $7.3 \pm 1.5$ & $13.58 \pm 4.38$ & [28] \\
\hline \multirow{2}{*}{$\begin{array}{l}\text { Hispaniolan } \\
\text { Parrot }\end{array}$} & \multirow{2}{*}{$\begin{array}{c}\text { Amazona } \\
\text { ventralis }\end{array}$} & $319 \pm 30 \mathrm{~g}$ & $30 \mathrm{mg} / \mathrm{kg}$ & Oral & Food withheld $2 \mathrm{~h}$ after administration & Suspension & $1.67 \pm 1.17$ & $23.48 \pm 6.96$ & $4.85 \pm 0.33$ & $2.7 \pm 0.38$ & \multirow{2}{*}{ [29-32] } \\
\hline & & $319 \pm 30 \mathrm{~g}$ & $5 \mathrm{mg} / \mathrm{kg}$ & Intravenous & Food withheld $2 \mathrm{~h}$ after administration & - & - & & $1.54 \pm 0.51$ & $2.55 \pm 0.85$ & \\
\hline Indian Peafowl & Pavo Cristatus & $4.1 \pm 0.4 \mathrm{~kg}$ & $7.5 \mathrm{mg} / \mathrm{kg}$ & Oral & Not fasted. Free access to food. & Suspension & $116.0 \pm 79.14$ & - & $1.68 \pm 0.41$ & $5.10 \pm 2.29$ & [33] \\
\hline Muscovy Duck & Carina moschata & $2.6 \pm 0.7 \mathrm{~kg}$ & $30 \mathrm{mg} / \mathrm{kg}$ & Oral & Food withheld $2 \mathrm{~h}$ before procedure & Suspension & $780 \pm 300$ & - & $3.95 \pm 0.60$ & $1.51 \pm 1.21$ & {$[7,34]$} \\
\hline \multicolumn{12}{|c|}{ HYDROMORPHONE } \\
\hline \multirow{2}{*}{$\begin{array}{l}\text { Orange-winged } \\
\text { Parrot }\end{array}$} & \multirow{2}{*}{$\begin{array}{l}\text { Amazona } \\
\text { amazonica }\end{array}$} & $402.6 \pm 42 \mathrm{~g}$ & $1 \mathrm{mg} / \mathrm{kg}$ & $\mathrm{IM}$ & \multirow{2}{*}{$\begin{array}{l}\text { Ad libitum access to water and } \\
\text { pelleted diet }\end{array}$} & - & $179.1 \pm 28.4$ & $97.6 \pm 61.1$ & $1.74 \pm 0.351$ & - & \multirow{2}{*}[38,39,87]{} \\
\hline & & $402.6 \pm 42 \mathrm{~g}$ & $1 \mathrm{mg} / \mathrm{kg}$ & Intravenous & & - & - & - & $1.45 \pm 0.270$ & - & \\
\hline $\begin{array}{c}\text { American } \\
\text { Kestrel }\end{array}$ & Falco sparverius & $115 \pm 6.2 \mathrm{~g}$ & $0.6 \mathrm{mg} / \mathrm{kg}$ & $\mathrm{IM}$ & $\begin{array}{l}\text { No access to food or water throughout } \\
\text { blood sample collection }\end{array}$ & - & 112.10 & 75 & 1.25 & - & {$[35,36]$} \\
\hline Cockatiel & $\begin{array}{l}\text { Nymphicus } \\
\text { hollandicus }\end{array}$ & $78.5-130.8 \mathrm{~g}$ & $0.6 \mathrm{mg} / \mathrm{kg}$ & IM & $\begin{array}{l}\text { Ad libitum access to water and } \\
\text { pelleted diet }\end{array}$ & - & 135.8 & - & 0.99 & - & [37] \\
\hline \multicolumn{12}{|c|}{ BUPRENORPHINE } \\
\hline \multirow{2}{*}{ Grey Parrot } & Psittacus & $300-500 \mathrm{~g}$ & $0.1 \mathrm{mg} / \mathrm{kg}$ & $\mathrm{IM}$ & - & - & 68.7 & - & $1.04 \pm 0.14$ & - & {$[40,41]$} \\
\hline & & $300-500 \mathrm{~g}$ & $0.1 \mathrm{mg} / \mathrm{kg}$ & Intravenous & & - & - & - & 1.04 & - & \\
\hline & & Not reported & $0.6 \mathrm{mg} / \mathrm{kg}$ & IM & & - & 242.9 & 94.9 & 1.54 & - & \\
\hline American & Falco sparverius & Not reported & $0.6 \mathrm{mg} / \mathrm{kg}$ & Intravenous & $\begin{array}{l}\text { Kestrels were fed killed, previously } \\
\text { frozen micer }\end{array}$ & - & - & - & 1.76 & - & {$[42-46]$} \\
\hline Kestrel & mo & Not reported & $1.8 \mathrm{mg} / \mathrm{kg}$ & $\mathrm{IM}$ & ad libitum access to water & Sustained release & 69.2 & - & 11.1 & - & 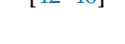 \\
\hline & & Not reported & $1.8 \mathrm{mg} / \mathrm{kg}$ & SC & & Sustained release & 72.3 & - & 13.5 & - & \\
\hline $\begin{array}{c}\text { Red-tailed } \\
\text { Hawk }\end{array}$ & Buteo jamaicensis & $1.28 \mathrm{~kg}$ & $0.3 \mathrm{mg} / \mathrm{kg}$ & SC & $\begin{array}{l}\text { Food withheld } 12 \mathrm{~h} \text { prior to } \\
\text { administration and normal feeding } \\
\text { schedule resume } 24 \mathrm{~h} \text { after }\end{array}$ & Concentrated & $74.1 \pm 37.0$ & - & $6.23 \pm 0.31$ & - & {$[46,47]$} \\
\hline & & $1.28 \mathrm{~kg}$ & $1.8 \mathrm{mg} / \mathrm{kg}$ & SC & administration & Concentrated & $322.1 \pm 57.7$ & - & $7.84 \pm 3.29$ & - & \\
\hline Cockatiel & $\begin{array}{l}\text { Nymphicus } \\
\text { hollandicus }\end{array}$ & $106 \pm 19.6 \mathrm{~g}$ & $0.6 \mathrm{mg} / \mathrm{kg}$ & $\mathrm{IM}$ & - & - & $240 \pm 44.3$ & - & 2.31 & - & [48] \\
\hline
\end{tabular}


Table 5. Cont.

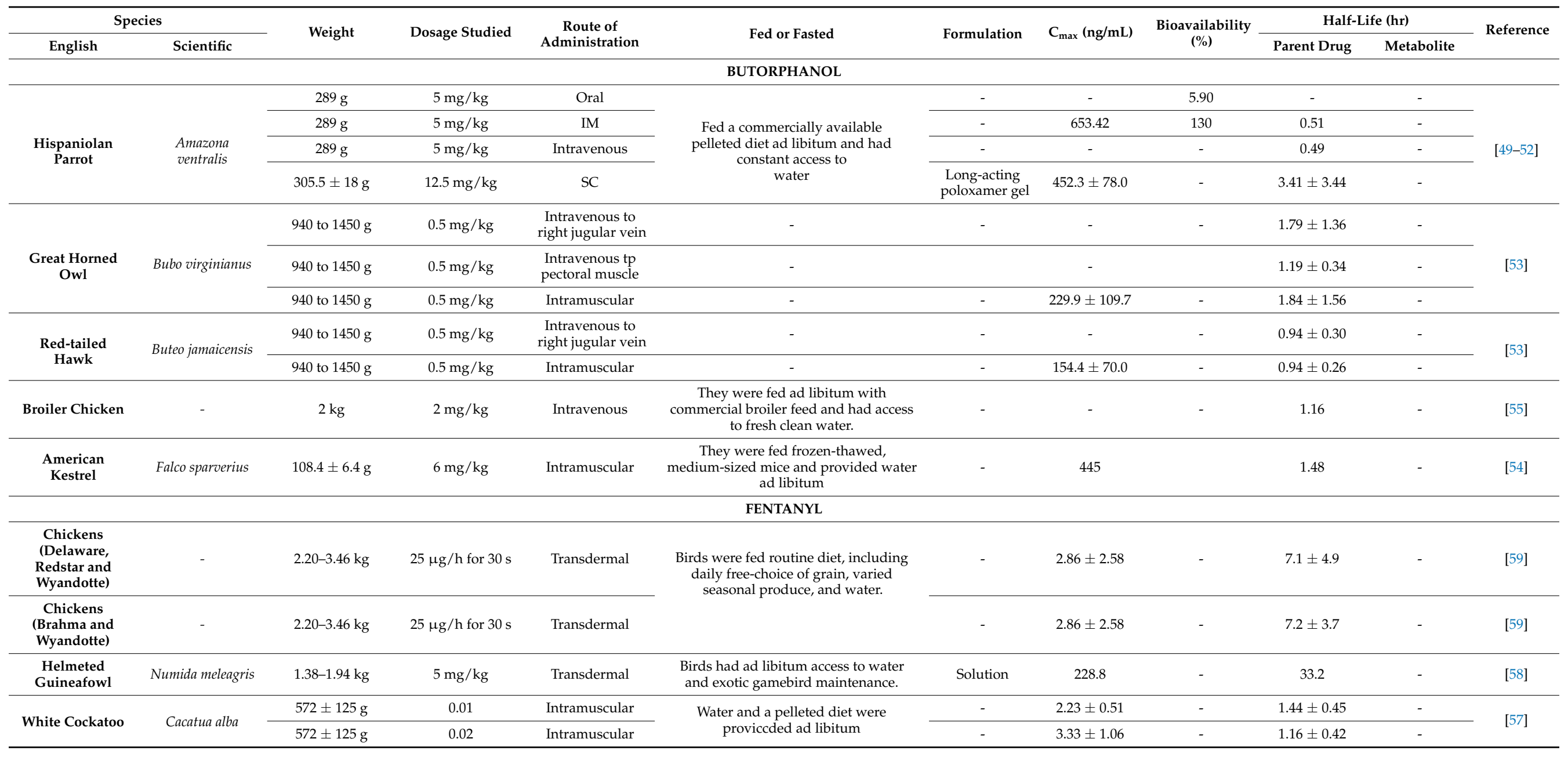




\subsection{Half-Life}

\subsubsection{Tramadol}

As compared to that of other species, tramadol's half-life is longest in African Penguin $(7.3 \pm 1.5 \mathrm{~h}) . \mathrm{M1}^{\prime} \mathrm{s}$ half-life is also longest in African Penguin (13.58 $\left.\pm 4.38 \mathrm{~h}\right)$ [28] although the weight of African Penguin in this study was comparable to other species. The reason for this is unknown although tramadol was administered with food in this study as compared to the other study design where food was not administered, although unrestricted. Presence of food usually increases transit time, which increases time for absorption. However, assuming linear PK, since half-life is generally affected by volume of distribution and clearance, presence of food is unlikely the reason for tramadol's long half-life in African Penguin.

Generally, Red-tailed Hawk and Hispaniolan Amazon have the shortest half-lives of around 1.3 to $1.5 \mathrm{~h}[25,27,29-32]$. Hispaniolan Amazon have the lowest weight among other species, which may explain its short half-life as weight may affect the volume of distribution and body size is correlated to basal metabolic rate. However, the reason for the shorter half-life of tramadol in red-tailed hawks is not clear.

In addition, the half-lives of tramadol in Hispaniolan Amazon were not consistent across the different routes of administration [29-32]. Its PO half-lives are higher than that of IV but it could be due to 6 times higher dose in PO as compared to IV. Conceptually, assuming linear PK, half-lives should be the same regardless of routes of administration and dose. This result may suggest tramadol, when administered to Hispaniolan Amazon, potentially exhibit saturable kinetics at higher doses. However, further study needs to be done to explain this observation.

\subsubsection{Hydromorphone}

The half-lives of hydromorphone in species studied range from $0.99-1.74 \mathrm{~h}$, with the longest half-life in Orange-winged Amazon and shortest half-life in Cockatiel $[38,39,50]$. This could be explained by their weights as cockatiel are the lightest whereas Orangewinged Amazon are the heaviest.

\subsubsection{Buprenorphine}

Generally, the half-lives of buprenorphine in species administered with standard buprenorphine formulation range from 1.04-2.31 h, with Cockatiel having the longest halflife among other species [48]. In this case, cockatiel are lighter than Grey Parrot, but have a longer-half life as compared to Grey Parrot [40,41]. This observation shows weight may not always be the basis of drug extrapolation, even among the same order of avian species.

\subsubsection{Butorphanol}

The half-lives of butorphanol differ between species studied. It ranges from $0.49-1.79 \mathrm{~h}$. Hispaniolan Amazon have the shortest half-life [49-52], which could be explained by its lightest weight. The Great Horned Owl appeared to have longer half-life than Red-tailed Hawk [53], which may be due to the presence of crops in Great Horned Owl that may withheld drug in the crop for a longer time.

\subsubsection{Fentanyl}

The half-lives of fentanyl in studied species range from 1.16-33.2 h. Helmeted Guineafowl have the longest half-life, which may be due to the long administration time of 7 days [58]. Other than that, there seems to be positive correlation between body weight and half-life, although no conclusion could be drawn yet given small number of species involved. 


\subsection{Bioavailability}

In general, the bioavailability of opioids was not reported in most studies. From the available data, it was observed that the bioavailability of opioids were generally high at around $75 \%$ or more, except that of Hispaniolan Amazon, where the oral bioavailability of tramadol and butorphanol were only $23.48 \%$ and $5.90 \%$, respectively [29-32,49-52].

For tramadol, among the species studied, Hispaniolan Amazon required a dose of $30 \mathrm{mg} / \mathrm{kg}$ to reach or exceed target plasma tramadol concentration in humans for up to $6 \mathrm{~h}$. This dose is generally higher than other species studied which could be due to lower tramadol bioavailability of $23.48 \%$ in parrots, as compared with $97.94 \%$ in eagles that only required $11 \mathrm{mg} / \mathrm{kg}$ oral tramadol to achieve the minimum effective tramadol concentration for up to $10 \mathrm{~h}$. A similar observation was made for butorphanol, where the bioavailability of oral butorphanol in Hispaniolan Amazon was only 5.90\%. The reason for this is unclear. Since there are no other studies available involving the use of oral butorphanol in other avian species, it is difficult to deduce whether Hispaniolan Amazon, as compared to other avian species, generally have low bioavailability for oral formulation of selected opioids, or bioavailability of butorphanol is generally low. In human, the bioavailability of oral butorphanol is very low (5\%) as it undergoes extensive first-pass metabolism [90]. Therefore, this could also possibly be the reason for the low oral bioavailability of butorphanol in birds.

Further research and study can be performed to assess the bioavailability of oral drugs in Hispaniolan Amazon compared to other bird species to explore bioavailability trend that may help advise oral dosing regimens in Hispaniolan Amazon.

\section{Conclusions}

This critical review found specific dosing regimens of tramadol for Indian peafowl, Muscovy Duck, and Hispaniolan Amazon [25,27,29-32]; hydromorphone for Orangewinged Amazon [38]; buprenorphine for Cockatiel, American Kestrel and Grey Parrot $[40,41,48]$; and butorphanol for Hispaniolan Amazon, Broiler Chicken and Indian Peafowl [49-52] which were not reported in the EAF. These regimens, if found to be effective based on the related PK and PD studies, could be used in practice.

In terms of efficacy, cockatiel did not seem to benefit from the analgesic effect of hydromorphone [50] and buprenorphine [48]. Furthermore, American Kestrel appeared to exhibit sex-dependent response to opioids [42-46]. More studies have to be performed to elucidate the reasons for these observations.

The use of these opioids were generally safe, with sedation being the most common adverse effect reported. Moderate adverse effects such as nausea-like behavior and GI adverse effects have been reported but no severe adverse effect such as death was seen. When using these opioids, one should monitor for these adverse effects, which are generally dose-dependent.

In terms of PK, an interesting observation was the lower bioavailability of oral tramadol in Hispaniolan Amazon compared to the bald eagle [29-32,49-52]. Additionally, low bioavailability of butorphanol was also observed in Hispaniolan Amazon. This could be due to the nature of butorphanol which has very low bioavailability in humans. Further research should be conducted to draw a firm conclusion on the oral bioavailability of drugs in Hispaniolan Amazon.

The main limitation of our work was that a systematic review approach was not undertaken. (Appendix B shows the items that this study fulfilled from the PRISMA checklist for systematic review). Hence, it may be possible that additional evidence of the PD and PK effects of the selected opioids may have been missed. Also, only publications in English were included. Our review highlights an urgent need for mechanistic studies to be performed to understand the underlying reasons for the variabilities observed. Coupled with more PD and PK studies in various avian species, a larger body of data may unveil any PD or PK trends that could then guide more accurate dose extrapolation to other bird species. 
Author Contributions: Conceptualization, S.X. and H.T.C.; Methodology, E.A., S.X. and H.T.C.; Investigation, E.A. and H.T.C.; Data curation, E.A.; Writing-original draft preparation, E.A. and H.T.C.; Writing-review and editing, H.T.C. and S.X.; Supervision-H.T.C. All authors have read and agreed to the published version of the manuscript.

Funding: This research received no external funding.

Institutional Review Board Statement: Not applicable.

Informed Consent Statement: Not applicable.

Data Availability Statement: Not applicable.

Acknowledgments: We sincerely thank the three anonymous reviewers for providing constructive feedback and comments which helped to improve this manuscript.

Conflicts of Interest: The authors declare no conflict of interest.

\section{Appendix A}

Table A1. Keywords used in search strategy.

\begin{tabular}{cc}
\hline Concept & Keywords \\
\hline Opioids & $\begin{array}{c}\text { Opioid, Opioids, Morphine, Buprenorphine, Butorphanol, Codeine, } \\
\text { Hydrocodone, Levorphanol, Meperidine, Tramadol, Methadone, Fentanyl, } \\
\text { Hydromorphone, Tapentadol, Oxymorphone, Oxycodone }\end{array}$ \\
\hline Bird & Bird, Birds, Aves, Avian \\
\hline $\begin{array}{c}\text { Pharmacokinetics } \\
\text { and Pharmacody- } \\
\text { namics }\end{array}$ & $\begin{array}{c}\text { PK or Pharmacokinetic or Pharmacokinetics or Disposition or Absorption } \\
\text { or Absorbed or Bioavailability or Biological Availability or AUC or Area } \\
\text { Under the Curve or Area-under-curve or Half life or Half-life or } \\
\text { Distribution or Distributed or Volume of distribution or Metabolism or } \\
\text { or Metabolic Inactivation or Excretion or Elimination or Clearance or } \\
\text { Dosing or Dosage or Dosage Regimen or Dosage Regimens or Therapeutic } \\
\text { concentration or Therapeutic concentrations or Therapeutic drug } \\
\text { concentration or Therapeutic drug concentrations or PD or }\end{array}$ \\
& $\begin{array}{c}\text { Pharmacodynamics or Pharmacodynamic or Analgesia or Analgesic or } \\
\text { Analgesics or Analgesic activity or Analgesic activities }\end{array}$ \\
\hline
\end{tabular}

\section{Appendix B}

Table A2. PRISMA checklist.

\begin{tabular}{|c|c|c|c|}
\hline Section and Topic & Item \# & Checklist Item & $\begin{array}{l}\text { Location Where } \\
\text { Item Is Reported }\end{array}$ \\
\hline \multicolumn{4}{|c|}{ TITLE } \\
\hline Title & 1 & Identify the report as a systematic review. & $\begin{array}{l}\text { NA because it is a critical, not } \\
\text { systematic, review }\end{array}$ \\
\hline \multicolumn{4}{|c|}{ ABSTRACT } \\
\hline Abstract & 2 & See the PRISMA 2020 for Abstracts checklist. & Abstract Line 21-35 \\
\hline \multicolumn{4}{|c|}{ INTRODUCTION } \\
\hline Rationale & 3 & $\begin{array}{l}\text { Describe the rationale for the review in the context of } \\
\text { existing knowledge. }\end{array}$ & Introduction Line 39-102 \\
\hline Objectives & 4 & $\begin{array}{l}\text { Provide an explicit statement of the objective(s) or } \\
\text { question(s) the review addresses. }\end{array}$ & Line 106-108 \\
\hline
\end{tabular}


Table A2. Cont.

\begin{tabular}{|c|c|c|c|}
\hline Section and Topic & Item \# & Checklist Item & $\begin{array}{l}\text { Location Where } \\
\text { Item Is Reported }\end{array}$ \\
\hline \multicolumn{4}{|c|}{ METHODS } \\
\hline Eligibility criteria & 5 & $\begin{array}{l}\text { Specify the inclusion and exclusion criteria for the review } \\
\text { and how studies were grouped for the syntheses. }\end{array}$ & $\begin{array}{l}\text { Method section (line 109-119). } \\
\text { Did not include exclusion } \\
\text { criteria. }\end{array}$ \\
\hline Information sources & 6 & $\begin{array}{l}\text { Specify all databases, registers, websites, organisations, } \\
\text { reference lists and other sources searched or consulted to } \\
\text { identify studies. Specify the date when each source was } \\
\text { last searched or consulted. }\end{array}$ & Method section line 110 \\
\hline Search strategy & 7 & $\begin{array}{l}\text { Present the full search strategies for all databases, registers } \\
\text { and websites, including any filters and limits used. }\end{array}$ & Search term in Appendix A \\
\hline Selection process & 8 & $\begin{array}{l}\text { Specify the methods used to decide whether a study met } \\
\text { the inclusion criteria of the review, including how many } \\
\text { reviewers screened each record and each report retrieved, } \\
\text { whether they worked independently, and if applicable, } \\
\text { details of automation tools used in the process. }\end{array}$ & NA \\
\hline Data collection process & 9 & $\begin{array}{l}\text { Specify the methods used to collect data from reports, } \\
\text { including how many reviewers collected data from each } \\
\text { report, whether they worked independently, any } \\
\text { processes for obtaining or confirming data from study } \\
\text { investigators, and if applicable, details of automation } \\
\text { tools used in the process. }\end{array}$ & $\begin{array}{l}\text { The first author } \\
\text { collected the data }\end{array}$ \\
\hline \multirow[t]{2}{*}{ Data items } & $10 \mathrm{a}$ & $\begin{array}{l}\text { List and define all outcomes for which data were sought. } \\
\text { Specify whether all results that were compatible with each } \\
\text { outcome domain in each study were sought (e.g., for all } \\
\text { measures, time points, analyses), and if not, the methods } \\
\text { used to decide which results to collect. }\end{array}$ & Method section line 116-119 \\
\hline & $10 \mathrm{~b}$ & $\begin{array}{l}\text { List and define all other variables for which data were } \\
\text { sought (e.g., participant and intervention characteristics, } \\
\text { funding sources). Describe any assumptions made about } \\
\text { any missing or unclear information. }\end{array}$ & NA \\
\hline $\begin{array}{l}\text { Study risk of bias } \\
\text { assessment }\end{array}$ & 11 & $\begin{array}{l}\text { Specify the methods used to assess risk of bias in the } \\
\text { included studies, including details of the tool(s) used, how } \\
\text { many reviewers assessed each study and whether they } \\
\text { worked independently, and if applicable, details of } \\
\text { automation tools used in the process. }\end{array}$ & NA \\
\hline Effect measures & 12 & $\begin{array}{l}\text { Specify for each outcome the effect measure(s) (e.g., risk } \\
\text { ratio, mean difference) used in the synthesis or } \\
\text { presentation of results. }\end{array}$ & NA \\
\hline \multirow[t]{2}{*}{ Synthesis methods } & $13 a$ & $\begin{array}{l}\text { Describe the processes used to decide which studies were } \\
\text { eligible for each synthesis (e.g., tabulating the study } \\
\text { intervention characteristics and comparing against the } \\
\text { planned groups for each synthesis (item \#5)). }\end{array}$ & NA \\
\hline & $13 b$ & $\begin{array}{l}\text { Describe any methods required to prepare the data for } \\
\text { presentation or synthesis, such as handling of missing } \\
\text { summary statistics, or data conversions. }\end{array}$ & $\begin{array}{l}\text { Conversion of units for some } \\
\text { of the data }\end{array}$ \\
\hline
\end{tabular}


Table A2. Cont.

\begin{tabular}{|c|c|c|c|}
\hline Section and Topic & Item \# & Checklist Item & $\begin{array}{l}\text { Location Where } \\
\text { Item Is Reported }\end{array}$ \\
\hline & $13 c$ & $\begin{array}{l}\text { Describe any methods used to tabulate or visually display } \\
\text { results of individual studies and syntheses. }\end{array}$ & NA \\
\hline & $13 d$ & $\begin{array}{l}\text { Describe any methods used to synthesize results and } \\
\text { provide a rationale for the choice(s). If meta-analysis was } \\
\text { performed, describe the model(s), method(s) to identify } \\
\text { the presence and extent of statistical heterogeneity, and } \\
\text { software package(s) used. }\end{array}$ & NA \\
\hline & $13 e$ & $\begin{array}{c}\text { Describe any methods used to explore possible causes of } \\
\text { heterogeneity among study results (e.g., subgroup } \\
\text { analysis, meta-regression). }\end{array}$ & NA \\
\hline & $13 \mathrm{f}$ & $\begin{array}{l}\text { Describe any sensitivity analyses conducted to assess } \\
\text { robustness of the synthesized results. }\end{array}$ & NA \\
\hline $\begin{array}{l}\text { Reporting bias } \\
\text { assessment }\end{array}$ & 14 & $\begin{array}{c}\text { Describe any methods used to assess risk of bias due to } \\
\text { missing results in a synthesis } \\
\text { (arising from reporting biases). }\end{array}$ & NA \\
\hline Certainty assessment & 15 & $\begin{array}{l}\text { Describe any methods used to assess certainty (or } \\
\text { confidence) in the body of evidence for an outcome. }\end{array}$ & NA \\
\hline
\end{tabular}

\section{RESULTS}

Describe the results of the search and selection process,

16a from the number of records identified in the search to the number of studies included in the review, ideally using a

Study selection flow diagram.

\begin{tabular}{|c|c|c|c|}
\hline & $16 \mathrm{~b}$ & $\begin{array}{l}\text { Cite studies that might appear to meet the inclusion } \\
\text { criteria, but which were excluded, and explain why they } \\
\text { were excluded. }\end{array}$ & NA \\
\hline Study characteristics & 17 & Cite each included study and present its characteristics. & NA \\
\hline Risk of bias in studies & 18 & Present assessments of risk of bias for each included study. & NA \\
\hline $\begin{array}{l}\text { Results of individual } \\
\text { studies }\end{array}$ & 19 & $\begin{array}{l}\text { For all outcomes, present, for each study: (a) summary } \\
\text { statistics for each group (where appropriate) and (b) an } \\
\text { effect estimate and its precision (e.g., confidence/credible } \\
\text { interval), ideally using structured tables or plots. }\end{array}$ & NA \\
\hline \multirow{4}{*}{ Results of syntheses } & $20 \mathrm{a}$ & $\begin{array}{l}\text { For each synthesis, briefly summarise the characteristics } \\
\text { and risk of bias among contributing studies. }\end{array}$ & NA \\
\hline & $20 \mathrm{~b}$ & $\begin{array}{l}\text { Present results of all statistical syntheses conducted. If } \\
\text { meta-analysis was done, present for each the summary } \\
\text { estimate and its precision (e.g., confidence/credible } \\
\text { interval) and measures of statistical heterogeneity. If } \\
\text { comparing groups, describe the direction of the effect. }\end{array}$ & NA \\
\hline & $20 c$ & $\begin{array}{l}\text { Present results of all investigations of possible causes of } \\
\text { heterogeneity among study results. }\end{array}$ & NA \\
\hline & $20 \mathrm{~d}$ & $\begin{array}{l}\text { Present results of all sensitivity analyses conducted to } \\
\text { assess the robustness of the synthesized results. }\end{array}$ & NA \\
\hline Reporting biases & 21 & $\begin{array}{l}\text { Present assessments of risk of bias due to missing results } \\
\text { (arising from reporting biases) for each synthesis assessed. }\end{array}$ & NA \\
\hline Certainty of evidence & 22 & $\begin{array}{l}\text { Present assessments of certainty (or confidence) in the } \\
\text { body of evidence for each outcome assessed. }\end{array}$ & NA \\
\hline
\end{tabular}


Table A2. Cont.

\begin{tabular}{|c|c|c|c|}
\hline Section and Topic & Item \# & Checklist Item & $\begin{array}{l}\text { Location Where } \\
\text { Item Is Reported }\end{array}$ \\
\hline \multicolumn{4}{|c|}{ DISCUSSION } \\
\hline \multirow{4}{*}{ Discussion } & $23 a$ & $\begin{array}{l}\text { Provide a general interpretation of the results in the } \\
\text { context of other evidence. }\end{array}$ & Sections 3-7 \\
\hline & $23 b$ & $\begin{array}{l}\text { Discuss any limitations of the evidence included in the } \\
\text { review. }\end{array}$ & NA \\
\hline & $23 c$ & Discuss any limitations of the review processes used. & Line $434-436$ \\
\hline & $23 \mathrm{~d}$ & $\begin{array}{l}\text { Discuss implications of the results for practice, policy, and } \\
\text { future research. }\end{array}$ & Section 8 Conclusion \\
\hline \multicolumn{4}{|c|}{ OTHER INFORMATION } \\
\hline \multirow{3}{*}{$\begin{array}{l}\text { Registration and } \\
\text { protocol }\end{array}$} & $24 a$ & $\begin{array}{l}\text { Provide registration information for the review, including } \\
\text { register name and registration number, or state that the } \\
\text { review was not registered. }\end{array}$ & Not registered \\
\hline & $24 b$ & $\begin{array}{l}\text { Indicate where the review protocol can be accessed, or } \\
\text { state that a protocol was not prepared. }\end{array}$ & Protocol not prepared \\
\hline & $24 \mathrm{c}$ & $\begin{array}{l}\text { Describe and explain any amendments to information } \\
\text { provided at registration or in the protocol. }\end{array}$ & NA \\
\hline Support & 25 & $\begin{array}{l}\text { Describe sources of financial or non-financial support for } \\
\text { the review, and the role of the funders or sponsors in the } \\
\text { review. }\end{array}$ & Under Funding statement \\
\hline Competing interests & 26 & Declare any competing interests of review authors. & $\begin{array}{l}\text { Under conflict of } \\
\text { interest declaration }\end{array}$ \\
\hline $\begin{array}{l}\text { Availability of data, } \\
\text { code and other materials }\end{array}$ & 27 & $\begin{array}{l}\text { Report which of the following are publicly available and } \\
\text { where they can be found: template data collection forms; } \\
\text { data extracted from included studies; data used for all } \\
\text { analyses; analytic code; any other materials used in the } \\
\text { review. }\end{array}$ & $\begin{array}{l}\text { All data presented } \\
\text { in the table can be found } \\
\text { in the references cited }\end{array}$ \\
\hline
\end{tabular}

\section{References}

1. Gill, F.; Donsker, D.; Rasmussen, P. IOC World Bird List (v11.1). Available online: https://www.worldbirdnames.org/new/ (accessed on 20 June 2021).

2. Number of Pet Birds in the U.S. 2017. Available online: https://www.statista.com/statistics/198107/birds-in-the-united-statessince-2000/ (accessed on 20 June 2021).

3. Singapore: Share of Pet Owners by Pet Type 2018. Available online: https://www.statista.com/statistics/1001965/singapore-petownership-rate-by-pet-type/ (accessed on 20 June 2021).

4. Hawkins, M.G. The use of analgesics in birds, reptiles, and small exotic mammals. J. Exot. Pet Med. 2006, 15, 177-192. [CrossRef]

5. Hawkins, M.G.; Paul-Murphy, J. Avian analgesia. Vet. Clin. N. Am. Exot. Anim. Pract. 2011, 14, 61-80. [CrossRef] [PubMed]

6. Robertson, S.A. Analgesia and analgesic techniques. Vet. Clin. N. Am. Exot. Anim. Pract. 2001, 4, 1-18. [CrossRef]

7. Bailey, R.S.; Sheldon, J.D.; Allender, M.C.; Adkesson, M.J.; Chinnadurai, S.K. Analgesic efficacy of tramadol compared with meloxicam in ducks (cairina moschata domestica) evaluated by ground-reactive forces. J Avian Med. Surg. 2019, 33, 133-140. [CrossRef]

8. Jenkins, J.R. Feather picking and self-mutilation in psittacine birds. Vet. Clin. N. Am. Exot. Anim. Pract. 2001, 4, 651-667. [CrossRef]

9. Lieberman, K.B.A.M. Feather-Picking in Parrots. Available online: https://www.vetmed.ucdavis.edu/sites/g/files/dgvnsk491 /files/inline-files/Feather-picking_in_Birds.pdf (accessed on 20 June 2021).

10. Hawkins, M.G.; Guzman, D.S.-M.; Beaufrère, H.; Lennox, A.M.; Carpenter, J.W. Chapter 5-Birds. In Exotic Animal Formulary, 5th ed.; Carpenter, J.W., Marion, C.J., Eds.; W.B. Saunders: Philadelphia, PA, USA, 2018; pp. 167-375.

11. Dorrestein, G.M.; van Miert, A.S. Pharmacotherapeutic aspects of medication of birds. J. Vet. Pharmacol. Ther. 1988, 11, 33-44. [CrossRef]

12. Lin, J.H. Applications and limitations of interspecies scaling and in vitro extrapolation in pharmacokinetics. Drug Metab. Dispos. $1998,26,1202-1212$. 
13. Mahmood, I. Application of allometric principles for the prediction of pharmacokinetics in human and veterinary drug development. Adv. Drug Deliv. Rev. 2007, 59, 1177-1192. [CrossRef]

14. Sharma, V.; McNeill, J.H. To scale or not to scale: The principles of dose extrapolation. Br. J. Pharmacol. 2009, 157, 907-921. [CrossRef]

15. Trescot, A.M.; Datta, S.; Lee, M.; Hansen, H. Opioid pharmacology. Pain Physician 2008, 11, S133-S153. [CrossRef]

16. Curro, T.G.; Brunson, D.B.; Paul-Murphy, J. Determination of the ED50 of isoflurane and evaluation of the isoflurane-sparing effect of butorphanol in cockatoos (Cacatua spp.). Vet. Surg. 1994, 23, 429-433. [CrossRef]

17. Curro, T.G. Evaluation of the isoflurane-sparing effects of butorphanol and flunixin in psittaciformes. In Proceedings of the Annual Conference of the Association of Avian Veterinarians, Reno, NV, USA, 28-30 September 1994; pp. 17-19.

18. Hawkins, M.G.; Pascoe, P.J.; DiMaio Knych, H.K.; Drazenovich, T.L.; Kass, P.H.; Sanchez-Migallon Guzman, D. Effects of three fentanyl plasma concentrations on the minimum alveolar concentration of isoflurane in Hispaniolan Amazon parrots (Amazona ventralis). Am. J. Vet. Res. 2018, 79, 600-605. [CrossRef]

19. Information, N.C.F.B. PubChem Compound Summary for CID 33741, Tramadol. Available online: https://pubchem.ncbi.nlm. nih.gov / (accessed on 20 June 2021).

20. National Center for Biotechnology Information. PubChem Compound Summary for CID 5284570, Hydromorphone. Available online: https:/ / pubchem.ncbi.nlm.nih.gov/compound/Hydromorphone (accessed on 20 June 2021).

21. National Center for Biotechnology Information. PubChem Compound Summary for CID 644073, Buprenorphine. Available online: https:/ / pubchem.ncbi.nlm.nih.gov/compound/Buprenorphine (accessed on 20 June 2021).

22. National Center for Biotechnology Information. PubChem Compound Summary for CID 5361092, Butorphanol. Available online: https:/ / pubchem.ncbi.nlm.nih.gov / compound/Butorphanol (accessed on 20 June 2021).

23. National Center for Biotechnology Information. PubChem Compound Summary for CID 3345, Fentanyl. Available online: https:/ / pubchem.ncbi.nlm.nih.gov/compound/Fentanyl (accessed on 20 June 2021).

24. Souza, M.J.; Martin-Jimenez, T.; Jones, M.P.; Cox, S.K. Pharmacokinetics of intravenous and oral tramadol in the bald eagle (Haliaeetus leucocephalus). J. Avian Med. Surg. 2009, 23, 247-252. [CrossRef]

25. Souza, M.J.; Cox, S.K. Tramadol use in zoologic medicine. Vet. Clin. N. Am. Exot. Anim. Pract. 2011, 14, 117-130. [CrossRef]

26. Guzman, D.S.; Drazenovich, T.L.; Olsen, G.H.; Willits, N.H.; Paul-Murphy, J.R. Evaluation of thermal antinociceptive effects after oral administration of tramadol hydrochloride to American kestrels (Falco sparverius). Am. J. Vet. Res. 2014, 75, 117-123. [CrossRef]

27. Souza, M.J.; Martin-Jimenez, T.; Jones, M.P.; Cox, S.K. Pharmacokinetics of oral tramadol in red-tailed hawks (Buteo jamaicensis). J. Vet. Pharmacol. Ther. 2011, 34, 86-88. [CrossRef]

28. Kilburn, J.J.; Cox, S.K.; Kottyan, J.; Wack, A.N.; Bronson, E. Pharmacokinetics of tramadol and its primary metabolite Odesmethyltramadol in African penguins (Spheniscus demersus). J. Zoo Wildl. Med. 2014, 45, 93-99. [CrossRef]

29. Sanchez-Migallon Guzman, D.; Souza, M.J.; Braun, J.M.; Cox, S.K.; Keuler, N.S.; Paul-Murphy, J.R. Antinociceptive effects after oral administration of tramadol hydrochloride in Hispaniolan Amazon parrots (Amazona ventralis). Am. J. Vet. Res. 2012, 73, 1148-1152. [CrossRef]

30. Souza, M.J.; Gerhardt, L.; Cox, S. Pharmacokinetics of repeated oral administration of tramadol hydrochloride in Hispaniolan Amazon parrots (Amazona ventralis). Am. J. Vet. Res. 2013, 74, 957-962. [CrossRef]

31. Geelen, S.; Sanchez-Migallon Guzman, D.; Souza, M.J.; Cox, S.; Keuler, N.S.; Paul-Murphy, J.R. Antinociceptive effects of tramadol hydrochloride after intravenous administration to Hispaniolan Amazon parrots (Amazona ventralis). Am. J. Vet. Res. 2013, 74, 201-206. [CrossRef]

32. Souza, M.J.; Sanchez-Migallon Guzman, D.; Paul-Murphy, J.R.; Cox, S.K. Pharmacokinetics after oral and intravenous administration of a single dose of tramadol hydrochloride to Hispaniolan Amazon parrots (Amazona ventralis). Am. J. Vet. Res. 2012, 73, 1142-1147. [CrossRef]

33. Black, P.A.; Cox, S.K.; Macek, M.; Tieber, A.; Junge, R.E. Pharmacokinetics of tramadol hydrochloride and its metabolite o-desmethyltramadol in peafowl (pavo cristatus). J. Zoo Wildl. Med. 2010, 41, 671-676. [CrossRef]

34. Bailey, R.S.; Sheldon, J.D.; Allender, M.C.; Papich, M.G.; Chinnadurai, S.K. Pharmacokinetics of orally administered tramadol in Muscovy ducks (Cairina moschata domestica). J. Vet. Pharmacol. Ther. 2019, 42, 380-384. [CrossRef]

35. Guzman, D.S.; Drazenovich, T.L.; Olsen, G.H.; Willits, N.H.; Paul-Murphy, J.R. Evaluation of thermal antinociceptive effects after intramuscular administration of hydromorphone hydrochloride to American kestrels (Falco sparverius). Am. J. Vet. Res. 2013, 74, 817-822. [CrossRef]

36. Guzman, D.S.; KuKanich, B.; Drazenovich, T.L.; Olsen, G.H.; Paul-Murphy, J.R. Pharmacokinetics of hydromorphone hydrochloride after intravenous and intramuscular administration of a single dose to American kestrels (Falco sparverius). Am. J. Vet. Res. 2014, 75, 527-531. [CrossRef]

37. Houck, E.L.; Guzman, D.S.; Beaufrère, H.; Knych, H.K.; Paul-Murphy, J.R. Evaluation of the thermal antinociceptive effects and pharmacokinetics of hydromorphone hydrochloride after intramuscular administration to cockatiels (Falco sparverius). Am. J. Vet. Res. 2018, 79, 820-827. [CrossRef]

38. Sanchez-Migallon Guzman, D.; Knych, H.; Douglas, J.; Paul-Murphy, J.R. Pharmacokinetics of hydromorphone hydrochloride after intramuscular and intravenous administration of a single dose to orange-winged Amazon parrots (Amazona amazonica). Am. J. Vet. Res. 2020, 81, 894-898. [CrossRef] 
39. Sanchez-Migallon Guzman, D.; Douglas, J.M.; Beaufrère, H.; Paul-Murphy, J.R. Evaluation of the thermal antinociceptive effects of hydromorphone hydrochloride after intramuscular administration to orange-winged Amazon parrots (Amazona amazonica). Am. J. Vet. Res. 2020, 81, 775-782. [CrossRef]

40. Paul-Murphy, J.; Hess, J.C.; Fialkowski, J.P. Pharmacokinetic Properties of a Single Intramuscular Dose of Buprenorphine in African Grey Parrots (Psittacus erithacus erithacus). J. Avian Med. Surg. 2004, 18, 224-228. [CrossRef]

41. Paul-Murphy, J.R.; Brunson, D.B.; Miletic, V. Analgesic effects of butorphanol and buprenorphine in conscious African grey parrots (Psittacus erithacus erithacus and Psittacus erithacus timneh). Am. J. Vet. Res. 1999, 60, 1218-1221.

42. Ceulemans, S.M.; Guzman, D.S.; Olsen, G.H.; Beaufrère, H.; Paul-Murphy, J.R. Evaluation of thermal antinociceptive effects after intramuscular administration of buprenorphine hydrochloride to American kestrels (Falco sparverius). Am. J. Vet. Res. 2014, 75, 705-710. [CrossRef] [PubMed]

43. Gustavsen, K.A.; Guzman, D.S.; Knych, H.K.; Petritz, O.A.; Olsen, G.H.; Paul-Murphy, J.R. Pharmacokinetics of buprenorphine hydrochloride following intramuscular and intravenous administration to American kestrels (Falco sparverius). Am. J. Vet. Res. 2014, 75, 711-715. [CrossRef] [PubMed]

44. Guzman, D.S.-M.; Knych, H.K.; Olsen Glenn, H.; Paul-Murphy, J.R. Pharmacokinetics of a sustained release formulation of buprenorphine after intramuscular and subcutaneous administration to American Kestrels (Falco sparverius). J. Avian Med. Surg. 2017, 31, 102-107. [CrossRef]

45. Guzman, D.S.-M.; Ceulemans, S.M.; Beaufrère, H.; Olsen, G.H.; Paul-Murphy, J.R. Evaluation of the thermal antinociceptive effects of a sustained-release buprenorphine formulation after intramuscular administration to American kestrels (Falco sparverius). J. Avian Med. Surg. 2018, 32, 1-7. [CrossRef]

46. Mazor-Thomas, J.E.; Mann, P.E.; Karas, A.Z.; Tseng, F. Pain-suppressed behaviors in the red-tailed hawk (Buteo jamaicensis). Appl. Anim. Behav. Sci. 2014, 152, 83-91. [CrossRef]

47. Gleeson, M.D.; Guzman, D.S.-M.; Knych, H.K.; Kass, P.H.; Drazenovich, T.L.; Hawkins, M.G. Pharmacokinetics of a concentrated buprenorphine formulation in red-tailed hawks (Buteo jamaicensis). Am. J. Vet. Res. 2018, 79, 13-20. [CrossRef]

48. Guzman, D.S.; Houck, E.L.; Knych, H.K.D.; Beaufrère, H.; Paul-Murphy, J.R. Evaluation of the thermal antinociceptive effects and pharmacokinetics after intramuscular administration of buprenorphine hydrochloride to cockatiels (Falco sparverius). Am. J. Vet. Res. 2018, 79, 1239-1245. [CrossRef]

49. Guzman, D.S.-M.; Flammer, K.; Paul-Murphy, J.R.; Barker, S.A.; Tully, T.N., Jr. Pharmacokinetics of butorphanol after intravenous, intramuscular, and oral administration in hispaniolan amazon parrots (Amazona ventralis). J. Avian Med. Surg. 2011, 25, 185-191. [CrossRef]

50. Sladky, K.; Krugner-Higby, L.; Meek-Walker, E.; Heath, T.; Paul-Murphy, J. Serum concentrations and analgesic effects of liposome-encapsulated and standard butorphanol tartrate in parrots. Am. J. Vet. Res. 2006, 67, 775-781. [CrossRef]

51. Laniesse, D.; Guzman, D.S.; Knych, H.K.; Smith, D.A.; Mosley, C.; Paul-Murphy, J.R.; Beaufrère, H. Pharmacokinetics of butorphanol tartrate in a long-acting poloxamer 407 gel formulation administered to Hispaniolan Amazon parrots (Amazona ventralis). Am. J. Vet. Res. 2017, 78, 688-694. [CrossRef]

52. Paul-Murphy, J.R.; Sladky, K.K.; Krugner-Higby, L.A.; Stading, B.R.; Klauer, J.M.; Keuler, N.S.; Brown, C.S.; Heath, T.D. Analgesic effects of carprofen and liposome-encapsulated butorphanol tartrate in Hispaniolan parrots (Amazona ventralis) with experimentally induced arthritis. Am. J. Vet. Res. 2009, 70, 1201-1210. [CrossRef] [PubMed]

53. Riggs, S.M.; Hawkins, M.G.; Craigmill, A.L.; Kass, P.H.; Stanley, S.D.; Taylor, I.T. Pharmacokinetics of butorphanol tartrate in red-tailed hawks (Buteo jamaicensis) and great horned owls (Bubo virginianus). Am. J. Vet. Res. 2008, 69, 596-603. [CrossRef] [PubMed]

54. Guzman, D.S.; Drazenovich, T.L.; KuKanich, B.; Olsen, G.H.; Willits, N.H.; Paul-Murphy, J.R. Evaluation of thermal antinociceptive effects and pharmacokinetics after intramuscular administration of butorphanol tartrate to American kestrels (Falco sparverius). Am. J. Vet. Res. 2014, 75, 11-18. [CrossRef] [PubMed]

55. Singh, P.; Johnson, C.; Gartrell, B.; Mitchinson, S.; Chambers, P. Papers: Pharmacokinetics of butorphanol in broiler chickens. Vet. Rec. 2011, 168, 588. [CrossRef]

56. Clancy, M.M.; KuKanich, B.; Sykes, J.M. Pharmacokinetics of butorphanol delivered with an osmotic pump during a seven-day period in common peafowl (Pavo cristatus). Am. J. Vet. Res. 2015, 76, 1070-1076. [CrossRef]

57. Hoppes, S.; Flammer, K.; Hoersch, K.; Papich, M.; Paul-Murphy, J. Disposition and Analgesic Effects of Fentanyl in White Cockatoos (Cacatua alba). J. Avian Med. Surg. 2003, 17, 124-130. [CrossRef]

58. Waugh, L.; Knych, H.; Cole, G.; D'Agostino, J. Pharmacokinetic evaluation of a long-acting fentanyl solution after transdermal administration in helmeted guineafowl (Numida meleagris). J. Zoo Wildl. Med. 2016, 47, 468-473. [CrossRef]

59. Delaski, K.M.; Gehring, R.; Heffron, B.T.; Negrusz, A.; Gamble, K.C. Plasma concentrations of fentanyl achieved with transdermal application in chickens. J. Avian Med. Surg. 2017, 31, 6-15. [CrossRef]

60. Scott, L.J.; Perry, C.M. Tramadol: A review of its use in perioperative pain. Drugs 2000, 60, 139-176. [CrossRef]

61. Smith, H.S.; Raffa, R.B.; Pergolizzi, J.V.; Taylor, R.; Tallarida, R.J. Combining opioid and adrenergic mechanisms for chronic pain. Postgrad. Med. 2014, 126, 98-114. [CrossRef]

62. Bamigbade, T.A.; Davidson, C.; Langford, R.M.; Stamford, J.A. Actions of tramadol, its enantiomers and principal metabolite, O-desmethyltramadol, on serotonin (5-HT) efflux and uptake in the rat dorsal raphe nucleus. Br. J. Anaesth. 1997, 79, 352-356. [CrossRef] 
63. Wu, W.N.; McKown, L.A.; Gauthier, A.D.; Jones, W.J.; Raffa, R.B. Metabolism of the analgesic drug, tramadol hydrochloride, in rat and dog. Xenobiotica 2001, 31, 423-441. [CrossRef]

64. Grond, S.; Sablotzki, A. Clinical pharmacology of tramadol. Clin Pharm. 2004, 43, 879-923. [CrossRef]

65. Grond, S.; Meuser, T.; Uragg, H.; Stahlberg, H.J.; Lehmann, K.A. Serum concentrations of tramadol enantiomers during patientcontrolled analgesia. Br. J. Clin. Pharmacol. 1999, 48, 254-257. [CrossRef]

66. Lehmann, K.A.; Kratzenberg, U.; Schroeder-Bark, B.; Horrichs-Haermeyer, G. Postoperative patient-controlled analgesia with tramadol: Analgesic efficacy and minimum effective concentrations. Clin. J. Pain 1990, 6, 212-220. [CrossRef]

67. Bao, Y.J.; Hou, W.; Kong, X.Y.; Yang, L.; Xia, J.; Hua, B.J.; Knaggs, R. Hydromorphone for cancer pain. Cochrane Database Syst. Rev. 2016, 10, 1-38. [CrossRef]

68. Ocaña, M.; Cendán, C.M.; Cobos, E.J.; Entrena, J.M.; Baeyens, J.M. Potassium channels and pain: Present realities and future opportunities. Eur. J. Pharmacol. 2004, 500, 203-219. [CrossRef]

69. Yaksh, T.L. Pharmacology and mechanisms of opioid analgesic activity. Acta Anaesthesiol. Scand. 1997, 41, 94-111. [CrossRef]

70. Machelska, H.; Celik, M.Ö. Advances in achieving opioid analgesia without side effects. Front. Pharmacol. 2018, 9, 1388. [CrossRef]

71. Walsh, S.L.; Eissenberg, T. The clinical pharmacology of buprenorphine: Extrapolating from the laboratory to the clinic. Drug Alcohol Depend. 2003, 70, S13-S27. [CrossRef]

72. Pick, C.G.; Peter, Y.; Schreiber, S.; Weizman, R. Pharmacological characterization of buprenorphine, a mixed agonist-antagonist with kappa 3 analgesia. Brain Res. 1997, 744, 41-46. [CrossRef]

73. Leander, J.D. Buprenorphine is a potent kappa-opioid receptor antagonist in pigeons and mice. Eur. J. Pharmacol. 1988, 151, 457-461. [CrossRef]

74. Picker, M.J. Kappa agonist and antagonist properties of mixed action opioids in a pigeon drug discrimination procedure. J. Pharmacol. Exp. Ther. 1994, 268, 1190-1198.

75. Buprenorphine Hydrochloride. American Hospital Formulary Service Drug Information; Board of Directors of the American Society of Hospital Pharmacists: Bethesda, MD, USA, 2003; pp. 2061-2069.

76. Roughan, J.V.; Flecknell, P.A. Buprenorphine: A reappraisal of its antinociceptive effects and therapeutic use in alleviating post-operative pain in animals. Lab. Anim. 2002, 36, 322-343. [CrossRef]

77. Flecknell, P.A.; Liles, J.H. Assessment of the analgesic action of opioid agonist-antagonists in the rabbit. J. Assoc. Vet. Anaesth. Great Br. Irel. 1990, 17, 24-29. [CrossRef]

78. Jablonski, P.; Howden, B.O. Oral buprenorphine and aspirin analgesia in rats undergoing liver transplantation. Lab. Anim. 2002, 36, 134-143. [CrossRef]

79. Rance, M.J. Animal and molecular pharmacology of mixed agonist-antagonist analgesic drugs. Br. J. Clin. Pharmacol. 1979, 7 (Suppl. S3), 281s-286s. [CrossRef]

80. Cowan, A.; Lewis, J.W.; Macfarlane, I.R. Agonist and antagonist properties of buprenorphine, a new antinociceptive agent. Br. J. Pharmacol. 1977, 60, 537-545. [CrossRef]

81. Cowan, A.; Doxey, J.C.; Harry, E.J. The animal pharmacology of buprenorphine, an oripavine analgesic agent. Br. J. Pharmacol. 1977, 60, 547-554. [CrossRef]

82. Watson, P.J.; McQuay, H.J.; Bullingham, R.E.; Allen, M.C.; Moore, R.A. Single-dose comparison of buprenorphine 0.3 and 0.6 mg i.v. given after operation: Clinical effects and plasma concentration. Br. J. Anaesth. 1982, 54, 37-43. [CrossRef]

83. Gaggermeier, B.H.J.; Schatzmann, U. Investigations on analgesia in domestic pigeons (C. livia, Gmel., 1789, var. dom.) using buprenorphine and butorphanol. Proc. Eur. Assoc. Avian Vet. 2003, 70-73.

84. Mansour, A.; Khachaturian, H.; Lewis, M.E.; Akil, H.; Watson, S.J. Anatomy of CNS opioid receptors. Trends Neurosci. 1988, 11, 308-314. [CrossRef]

85. Commiskey, S.; Fan, L.W.; Ho, I.K.; Rockhold, R.W. Butorphanol: Effects of a prototypical agonist-antagonist analgesic on kappa-opioid receptors. J. Pharmacol. Sci. 2005, 98, 109-116. [CrossRef] [PubMed]

86. Chang, K.J.; Hazum, E.; Cuatrecasas, P. Novel opiate binding sites selective for benzomorphan drugs. Proc. Natl. Acad. Sci. USA 1981, 78, 4141-4145. [CrossRef]

87. Comer, S.D.; Cahill, C.M. Fentanyl: Receptor pharmacology, abuse potential, and implications for treatment. Neurosci. Biobehav. Rev. 2019, 106, 49-57. [CrossRef]

88. Fousse, S.L.; Golsen, B.M.; Sanchez-Migallon Guzman, D.; Paul-Murphy, J.R.; Stern, J.A. Varying Expression of Mu and Kappa Opioid Receptors in Cockatiels (Falco sparverius) and Domestic Pigeons (Columba livia domestica). Front. Genet. 2020, 11, 1128. [CrossRef]

89. Guigueno, M.F.; Karouna-Renier, N.K.; Henry, P.F.P.; Head, J.A.; Peters, L.E.; Palace, V.P.; Letcher, R.J.; Fernie, K.J. Female hatchling American kestrels have a larger hippocampus than males: A link with sexual size dimorphism? Behav. Brain Res. 2018, 349, 98-101. [CrossRef]

90. Butorphanol Tartrate Summary Report; The European Agency for the Evaluation of Medicinal Products Veterinary Medicines Evaluation Unit: London, UK, January 1998. 